\title{
Multi-dimensional signaling method for population-based metaheuristics: solving the large-scale scheduling problem in smart grids
}

Author: João Soares (Corresponding author)

Affiliation: GECAD - Research Group on Intelligent Engineering and Computing for Advanced Innovation and Development - Polytechnic of Porto (IPP)

Address: R. Dr. António Bernardino de Almeida, 431, 4200-072 Porto, Portugal

Email: joaps@isep.ipp.pt

Author: M. Ali. Fotouhi

Affiliation: GECAD - Research Group on Intelligent Engineering and Computing for Advanced Innovation and Development - Polytechnic of Porto (IPP)

Address: R. Dr. António Bernardino de Almeida, 431, 4200-072 Porto, Portugal

Email: mafgh@isep.ipp.pt

Author: Marco Silva

Affiliation: GECAD - Research Group on Intelligent Engineering and Computing for Advanced Innovation and Development - Polytechnic of Porto (IPP)

Address: R. Dr. António Bernardino de Almeida, 431, 4200-072 Porto, Portugal

Email: masi@isep.ipp.pt

Author: Zita Vale

Affiliation: GECAD - Research Group on Intelligent Engineering and Computing for Advanced Innovation and Development - Polytechnic of Porto (IPP)

Address: R. Dr. António Bernardino de Almeida, 431, 4200-072 Porto, Portugal

Email: zav@isep.ipp.pt 


\title{
Multi-dimensional signaling method for population-based metaheuristics: solving a large-scale scheduling problem in smart grids
}

\author{
João Soares ${ }^{1}$, M. Ali Fotouhi' ${ }^{1}$, Marco Silva' ${ }^{1}$ Zita Vale $^{1}$ \\ ${ }^{1}$ GECAD - Research Group on Intelligent Engineering and Computing for Advanced \\ Innovation and Development - Polytechnic of Porto (IPP), R. Dr. António Bernardino de \\ Almeida, 431, 4200-072 Porto, Portugal \\ * Corresponding author: João Soares \\ Tel.: +351 22 8340500; Fax: +351 22 8321159, joaps@isep.ipp.pt
}

\section{ABSTRACT}

The dawn of smart grid is posing new challenges to grid operation. The introduction of Distributed Energy Resources (DER) requires tough planning and advanced tools to efficiently manage the system at reasonable costs. Virtual Power Players (VPP) are used as means of aggregating generation and demand, which enable smaller producers using different generation technologies to be more competitive. This paper discusses the problem of the centralized Energy Resource Management (ERM), including several types of resources, such as Demand Response (DR), Electric Vehicles (EV) and Energy Storage Systems (ESS) from the VPP's perspective to maximize profits. The complete formulation of this problem, which includes the network constraints, is represented with a complex large-scale mixed integer nonlinear problem. This paper focuses on deterministic and metaheuristics methods and proposes a new multi-dimensional signaling approach for population-based random search techniques. The new approach is tested with two networks with high penetration of DERs. The results show outstanding performance with the proposed multi-dimensional signaling and confirm that standard metaheuristics are prone to fail in solving these kind of problems.

KEYWORDS: Large-scale Nonlinear Optimization; Metaheuristics; Particle Swarm Optimization; Smart Grid Management; Swarm Intelligence 


\section{Introduction}

The advent of Smart Grid (SG) over the past few years is being possible due to major technological breakthroughs, namely in Information and Communications Technology (ICT) and increased penetration of Distributed Generation (DG) of several types [1]. These new resources require new methods for optimal management of resources in distribution systems. In this context new algorithms are needed for the SG, namely for addressing the Energy Resource Management (ERM) problems, which is the focus of this paper.

In the recent years, several studies have been focused on the ERM in SGs; moreover, the increasing number of Electric Vehicles (EVs) have gave rise to several research opportunities in the ERM field. The works reported in $[2,3]$ present a unit commitment model with gridable EVs using Particle Swarm Optimization (PSO) to reduce costs and emissions in SGs increasing the security and reliability of utility grids. These works only use PSO to solve the optimization problem without comparing with other methods, namely other metaheuristics or deterministic approaches. Works reported in [4,5] and [6] present a PSO and a simulated annealing technique, respectively, to solve the day-ahead Distributed Energy Resources (DER) problem using a single-objective function. Both works compare the use of the metaheuristic with a conventional mathematical method demonstrating that the use of metaheuristics can be interesting to reduce the computational burden while preserving good solutions. The influence of the successive day on the day-ahead optimal solution is discussed in [7]. Authors in [8] develop an expert management system of a MicroGrid (MG) with the aim to minimize the operation costs and emissions of the MG operation by using a modified bacteria foraging algorithm. The model considers a simple load balance (active power) and does not consider the presence of EVs neither any type of Demand Response (DR). In [9] an intelligent energy management for a MG using intelligent techniques and linear-programming is presented. The model aims at minimizing operation cost and environmental impact. The formulation does not consider the network constraints, EVs, DR, and Energy Storage Systems (ESS), which may not sound realistic. In [10], an optimization model for the energy scheduling of islanded power systems is developed. High costs of traditional generation motivate the introduction of ESS for improving the utilization of renewable generation. The model seems to demonstrate that ESS can be used to reduce electricity costs and improve the utilization of wind power. However, the model is only adequate for insular systems and does not consider the presence of EVs and flexible demand. A decentralized energy and reserve scheduling model is proposed in $[11,12]$ for the operation of smart grids. The model considers that the EVs provide the reserve requirements for compensating renewable generation variations while an intelligent parking lot is used to 
aggregate the stored energy of EVs. The model considers flexibility from EVs, but not from regular load. The work presented in [13] regards a two-stage stochastic formulation to address the energy scheduling in MG with DG, EVs and ESS. The model solves the day-ahead energy scheduling using a linear formulation without network constraints and not considering Vehicle-To-Grid (V2G). An iterative approach is used to validate the network constraints with a power flow software returned from the master linear problem. Several scenarios were considered only for wind and solar power. The EVs, load demand and grid hourly prices are considered deterministically.

The problem addressed in this work concerns the day-ahead ERM with the aim to support the operational decisions of Virtual Power Player (VPP) in the SG context. The VPP aims to manage the available resources as efficiently as possible with the objective of maximizing profits, namely by reducing the need to buy energy from the day-ahead market or from other external suppliers at high prices. The day-ahead ERM is performed the day before the energy transactions are due. Typically the considered transactions periods are one-hour long corresponding to 24 scheduling periods. An important input of the ERM is the demand forecast for each hour, which can be done using modern accurate forecasting methods [8,14]. VPP can aggregate EVs and DR [15-18], hence it is important to consider the EVs' location in the grid and the respective energy demand throughout the day. The use of V2G, and ESS technology can help to increase the penetration of non-dispatchable intermittent renewable based DGs [19-21]. In this paper, ESS and EVs with V2G support are considered for this reason. V2G seems a promising technology and at least one commercial EV already supports V2G functions [22].

Due to the nature of the problem, ERM is generally classified as a large-scale combinational problem with integer variables and nonlinear features, e.g. AC network power balance. Nonconvex nonlinear and mixedinteger nonlinear NP-hard problems are difficult to solve with mathematical approaches but are suitable to be addressed by evolutionary computation [23,24]. The class of problems called Mixed Integer Nonlinear Programming (MINLP) is not only empirically more challenging than Mixed Integer Programing (MILP), but also theoretically harder [25]. It is possible to reduce the MINLP problem to MILP, e.g. without network constraints and/or quadratic cost functions, but, even when restricting the nonlinearity to linear or quadratic functions we know that the continuous relaxation of a MIQP is a NP-hard while the MILP are "only" NPcomplete [25]. A consequence of the definition of NP-hard is that if an optimization problem $H$ has an NPcomplete decision version $L$, then $H$ is NP-hard. Several similar problems in the literature have been reported as 
NP-hard related with smart grid problems [26-28]. Several metaheuristics have emerged in the last years and new methods continue to be proposed in the literature [29-32]. Many population-based metaheuristics are available, such as PSO and relatively new Differential Search Algorithm (DSA) [33]. In spite of their wide success some hard problems like the one discussed in this paper sometimes still very difficult to solve even with metaheuristics [34]. While some metaheuristics can perform better than others they largely depend in the setup and the problem to be optimized. The performance of a population-based random search technique lies in its global search ability, convergence speed, robustness, etc. [35]. Some authors agree that DSA is good at exploring the search space and locating the region of global minimum but it is slow at the exploitation of the solution presenting slow convergence rate [36]. Improvements in PSO algorithm have been made to overcome its premature convergence, e.g. Quantum-PSO (QPSO) presents better global convergence properties than PSO [35]. Nevertheless, in this work it is demonstrated that even the latest metaheuristics are prone to fail to solve the problem under study, thus requiring further research and robust mechanisms to handle this kind of optimization problem.

Hence, a new vision is proposed to implement metaheuristics with the aim to solve hard large-scale combinatorial problems more efficiently. An improved signaling mechanism compatible with any populationbased metaheuristic is proposed. This work has roots in [34,37] but the signaling mechanism previously developed is only applicable to PSO with velocity vectors and thus more limited. The new signaling mechanism, called multi-dimensional signaling, is tested with Quantum-based Particle Swarm Optimization (QPSO) [35], a new meta-heuristic called DSA [33] and PSO [38] for the ERM large-scale problem and can be adapted to other metaheuristic and problems. The proposed mechanism is new and not found in the literature. For stronger analysis two mathematical approaches are developed in this work namely a linear relaxed formulation using Mixed-Integer Linear Programming (MILP) implemented in TOMLAB [39] and a full nonlinear formulation (full network constraints) using Mixed-Integer Nonlinear Programming (MINLP) in GAMS [40]. Furthermore a hybrid version combining deterministic and DSA is developed. These approaches are compared with the mentioned metaheuristics. To test the effectiveness of the methods, a case study is developed using two different distribution networks, namely a $12 \mathrm{kV}$ network with 33-bus and a larger $30 \mathrm{kV}$ network with 180 -bus, to represent realistic scenarios with high penetration of DG and EVs. 
This paper is organized as follows: Section 2 describes the adopted control architecture of a VPP. Section 3 presents the mathematical model of the ERM problem. Section 4 presents the proposed multi-dimensional signaling method. Section 5 briefly discusses the used methods. Section 6 depicts the case study and the discussion of the obtained results. Finally, the most relevant conclusions are duly drawn in Section 7.

\section{Control architectures of VPP}

The number of Distributed Energy Resources (DER) is growing fast. Running a high number of DER under market conditions is inevitable but there are some few issues to consider. DER are not able to participate in the current markets due to their small size; their intermittent nature (wind and solar units) limits their contribution to the grid operation causing economic penalties as a result of unexpected unbalances; and different ownerships limit the cooperation and communication between neighboring units. A possible way to mitigate the issues inherent to DER penetration is to aggregate the dispersed units in a Virtual Power Player (VPP). The aggregation enables the same visibility, controllability and market functionality as conventional generation [41]. VPPs can emerge by geographical location, functional needs, generation technologies, etc. The control strategy of VPP can be classified into three categories: centralized control, decentralized and fully decentralized control [41,42]. Centralized controlled VPP is a control strategy that requires complete knowledge of involved DER units as well as every operating set point to meet the local requirements [6,43]. Decentralized controlled VPP is a collection of distributed small local controllers constituting a hierarchical architecture. In this control strategy a central controller is required to ensure system security and overall economic operation [44]. Fully decentralized controlled VPP provides valuable information such as market price, weather forecasting and data logging to DER participants where each node can have local control [41]. Fig. 1 shows the three types of control strategies described before. 


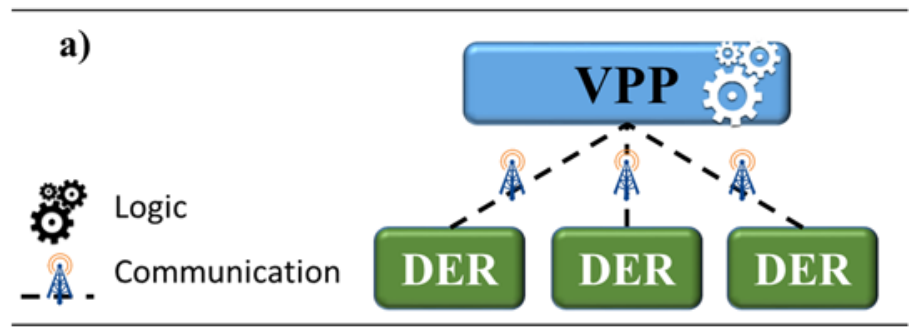

b)

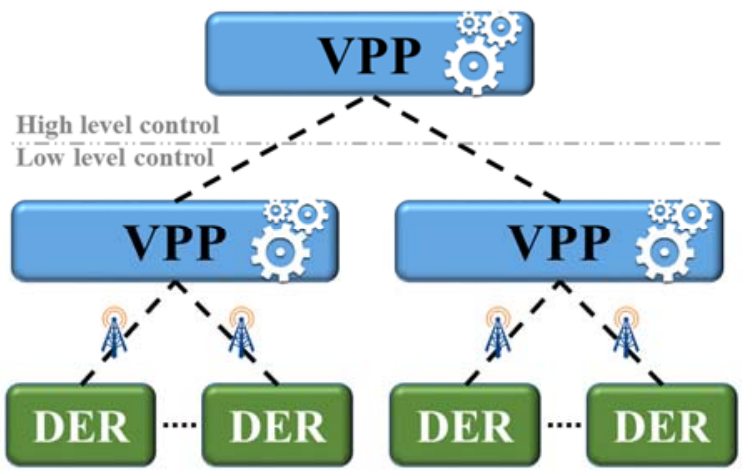

c)

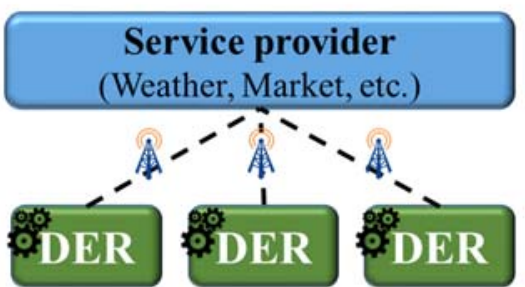

Fig. 1. VPP control strategies: a) centralized b) decentralized c) fully decentralized (adapted from [42]).

In this paper the centralized approach is chosen, which can be specific of some VPP but it is the most complex control strategy that requires reliable communications, control mechanisms and adequate computing processing due to the large-scale combinatorial optimization. However, centralized approach has a high potential of reaching optimal operation $[41,45]$. It is assumed that the VPP is responsible for the distribution network in its area in this study, which is equipped with information and communication means that enable the VPP to monitor and actively control the network. The optimization problem is of large-scale due to the high number of decision variables resulting from the high penetration of distributed energy resources and necessary information involved.

\section{Energy resources management mathematical model}

The VPP aims to maximize profits, i.e. minimize the total operation costs (1) over the entire $T$ periods and maximize the income (2) over the entire $T$ periods. This ERM problem is a hard combinatorial MINLP problem, due to the high number of continuous and discrete variables and the nonlinear network equations. The decision variables of the optimization are the generation powers of the energy resources, the commitment of the DG units, 
ESSs and the EVs' charge/discharge schedules, the loads' demand response for each unit/load and each period, as well as the voltage and angles in each bus during the scheduling period. It is a multi-period optimization with $T$ periods.

\subsection{Model assumptions}

The ERM problem is developed based on the following assumptions:

- The model relies on the high accuracy of load forecasts. It is assumed that the VPP can predict the demand profile with small errors by implementing the models developed in $[8,13]$.

- The VPP is equipped with adequate infrastructure for actively controlling the network, monitoring its conditions and guaranteeing the optimal operation of the grid.

- The discharging price of ESSs and EVs cover the battery degradation costs and remunerates the discharging service.

- The VPP is able to sell energy to the main grid and external entities.

\subsection{Objective function}

The multi-objective optimization model includes the following two objective functions:

$$
\begin{aligned}
& \text { Minimize } O C_{\text {Total }}^{D+1}=
\end{aligned}
$$

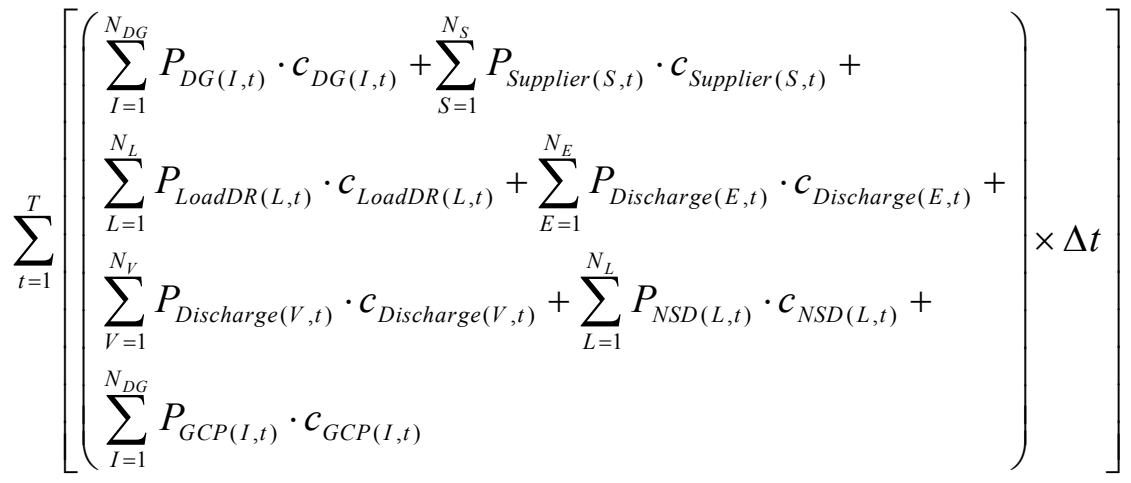

$$
\begin{aligned}
& \text { Maximize } I_{\text {Total }}^{D+1}= \\
& \sum_{t=1}^{T}\left[\left(\begin{array}{l}
\sum_{L=1}^{N_{L}} P_{\text {Load }(L, t)} \cdot M P_{\text {Load }(L, t)}+\sum_{M=1}^{N_{M}} P_{\operatorname{Sell}(M, t)} \cdot M P_{\text {Sell }(M, t)}+ \\
\sum_{E=1}^{N_{E}} P_{\text {Charge }(E, t)} \cdot M P_{\text {Charge }(E, t)}+\sum_{V=1}^{N_{V}} P_{\text {Charge }(V, t)} \cdot M P_{\text {Charge }(V, t)}
\end{array}\right) \times \Delta t\right]
\end{aligned}
$$


The objective function (1) represents the minimization of the electricity acquisition costs. The first term corresponds to the generation cost of the DG units, such as PV, wind and biomass units. The second term includes the cost of providing electricity by the external suppliers. The costs of implementing DR programs is reflected in the third term. The costs associated with the discharging of EVs and ESSs are respectively shown in the fourth and fifth terms. The last two terms in (1) correspond to the penalizations with non-supplied demand (NSD) and generation curtailment (GCP) due to excess power.

The objective function (2) concerns the income obtained from selling electricity to loads and to the pool market. It also incorporates tge income from charging the EVs and the ESS. As both objective functions (1) and (2) represent the energy costs (m.u.), the multi-objective optimization problem can be rewritten as a single objective problem (3):

$$
\text { Maximize } f=I_{\text {Total }}^{D+1}-O C_{\text {Total }}^{D+1}
$$

or

$$
\text { Minimize } f=O C_{\text {Total }}^{D+1}-I_{\text {Total }}^{D+1}
$$

\subsection{Constraints}

Objective functions ( 3 ) is subject to the following constraints:

- The nodal active (4) and reactive (5) power balance constraints in power grids, considering the power loss in each period $t$ are as follows:

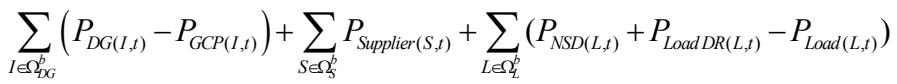

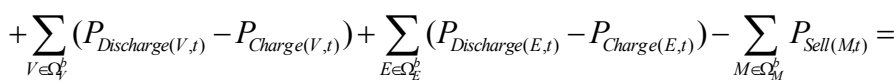

$$
\begin{aligned}
& \sum_{k=1}^{N_{B}} V_{b(t)} \cdot V_{k(t)} \cdot\left(G_{b k} \cdot \cos \left(\theta_{b(t)}-\theta_{k(t)}\right)+B_{b k} \cdot \sin \left(\theta_{b(t)}-\theta_{k(t)}\right)\right) \quad \forall t ; \forall b, k \neq b \\
& \sum_{I \in S_{L G G}^{b}} Q_{D G(I, t)}+\sum_{S \in \Omega_{G}^{b}} Q_{\text {Supplier }(S, t)}-\sum_{L \in \Omega_{L}^{b}} Q_{\text {Load }(L, t)}= \\
& \sum_{k=1}^{N_{B}} V_{b(t)} \times V_{k(t)}\left(G_{b k} \sin \left(\theta_{b(t)}-\theta_{k(t)}\right)-B_{b k} \cos \left(\theta_{b(t)}-\theta_{k(t)}\right)\right) \quad \forall t ; \forall b, k \neq b
\end{aligned}
$$

The right-hand side of constraints (4) and (5) correspond to the active and reactive power injected from bus $b$ into the grid.

- Bus voltage magnitude and angle levels at each bus are respectively represented by (6) and (7), assuming that the maximum and minimum limits remain fixed across the optimization horizon. 


$$
\begin{array}{ll}
V_{b}^{\text {min }} \leq V_{b(t)} \leq V_{b}^{\text {max }} & \forall t, \forall b \\
\theta_{b}^{\text {nin }} \leq \theta_{b(t)} \leq \theta_{b}^{\text {max }} & \forall t, \forall b
\end{array}
$$

- The apparent power flow in each line is constrained with the thermal line limits:

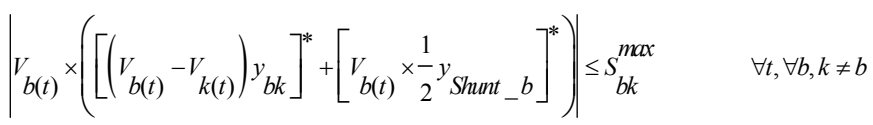

- HV/MV power transformers limits considering the power flow direction from HV to MV is shown in constraint (9).

$$
\sqrt{\left(\sum_{S \in \Omega_{S}^{b}} P_{\text {Suppier }(S, t)}\right)^{2}+\left(\sum_{S \in \Omega_{S}^{b}} Q_{\text {Supplier }(S, t)}\right)^{2}} \leq S_{\text {TFR_HV/MV }(b)}^{\max } \quad \forall t, \forall b
$$

- $\quad \mathrm{MV} / \mathrm{LV}$ power transformers limits are presented in constraints (10)-(12).

$$
\begin{aligned}
& P_{T F R \_M V / L V(b, t)}=\sum_{I \in S_{D G}^{p}}\left(P_{D G G(I, t)}-P_{G C P(I, t)}\right)+\sum_{L \in S_{L}^{b}}\left(P_{N S D(L, t)}+P_{\text {Load DRR }(L, t)}-P_{\text {Load }(L, t)}\right) \\
& +\sum_{V \in \Omega_{i}^{b}}\left(P_{D i s c h a r g e(V, t)}-P_{C h a r g e(V, t)}\right)+\sum_{E \in S_{E}^{b}}\left(P_{D i s c h a r g e(E, t)}-P_{\text {Charge }(E, t)}\right) \quad \forall t, \forall b \\
& Q_{T F R_{-} M V / L V(b, t)}=\sum_{I \in S_{D G}^{b}}\left(Q_{D G(I, t)}\right)-\sum_{L \in \Omega_{L}^{b}}\left(Q_{\text {Load }(L, t)}\right) \quad \forall t, \forall b \\
& \sqrt{\left(P_{T F R_{-} M V / L V(b, t)}^{2}+Q_{T F R_{-} M V / L V(b, t)}^{2}\right)} \leq S_{\text {TFR_MV/LV(b) }}^{\max } \quad \forall t, \forall b
\end{aligned}
$$

- The active and reactive power generation levels of the DG units during the online status are respectively constrained with (13) and (14):

$X_{D G(I, t)} \cdot P_{D G M n L i m i t(I, t)} \leq P_{D G(I, t)} \leq X_{D G(I, t)} \cdot P_{D G \text { MoxLinit }(I, t)} \quad \forall t, \forall I$

$X_{D G(I, t)} \times Q_{D G \operatorname{MinLinit}(I, t)} \leq Q_{D G(I, t)} \leq X_{D G(I, t)} \times Q_{D G \operatorname{MaxLimit}(I, t)} \quad \forall t, \forall I$

- The production of the upstream supplier is constrained with (15) and (16).

$P_{\text {Supplier }(S, t)} \leq X_{\text {Supplier }(S, t)} \cdot P_{\text {SupplierLimit }(S, t)} \quad \forall t, \forall S$

$Q_{\text {Sipplier }(S, t)} \leq X_{\text {Supplier(S,t)}} \cdot Q_{\text {SupplierLimit }(S, t)} \quad \forall t, \forall S$

- EVs cannot charge or discharge simultaneously. Constraint (17) imposes this condition to the ERM problem.

$X_{(V, t)}+Y_{(V, t)} \leq 1 \quad \forall t, \forall V$ 
- The relationship between the stored energy in EV $V$, the energy consumed during the trips and the charging/discharging power in each period $t$ is represented by constraint (18).

$E_{\text {Stored }(V, t)}=E_{\text {Stored }(V, t-1)}-E_{\text {Trip }(V, t)}+$

$\eta_{c(V)} \cdot P_{\text {Charge }(V, t)} \cdot \Delta t-\frac{1}{\eta_{d(V)}} \cdot P_{\text {Discharge }(V, t)} \cdot \Delta t$

$\forall t \in\{1, \ldots, T\} ; \quad \forall V \in\left\{1, \ldots, N_{V}\right\} ;$

- The maximum charging and discharging rates of EV $V$, when the EV is connected to grid, are respectively considered with constrinats (19) and (20).

$P_{\text {Charge }(V, t)} \leq P_{\text {ChargeLimit }(V, t)} \cdot Y_{(V, t)} \quad \forall t, \forall V$

$P_{\text {Discharge }(V, t)} \leq P_{\text {DischargeLimit }(V, t)} \cdot X_{(V, t)} \quad \forall t, \forall V$

- The charging power of the EV in time period $t$ should be calculated based on the capcity of the EV's battery and the energy stored in the battery by the end of time period $t-1$ (21).

$\eta_{c(V)} \cdot P_{\text {Charge }(V, t)} \cdot \Delta t \leq E_{\text {BatCap }(V)}-E_{\text {Stored }(V, t-1)} \quad \forall t, \forall V, \Delta t=1$

- EV discharge limit considering the battery balance. The vehicle cannot discharge more than the available energy in the battery. The discharging power of the EV in time period $t$ can not exceed the energy stored in the EV's battery by the end of time period $t-1$ (22).

$\frac{1}{\eta_{d(V)}} \cdot P_{\text {Discharge }(V, t)} \cdot \Delta t \leq E_{\text {Stored }(V, t-1)} \quad \forall t, \forall V, \Delta t=1$

- $\quad$ Battery capacity limit for each EV is considered in constraint (23).

$E_{\text {Stored }(V, t)} \leq E_{B a t C a p(V)} \quad \forall t, \forall V$

- Minimum stored energy to be guaranteed at the end of period $t$. This can be seen as a reserve energy (fixed by the EVs' users) that can be used for a regular travel or an unexpected travel in each period:

$E_{\text {Stored }(V, t)} \geq E_{\text {MinCharge }(V, t)} \quad \forall t, \forall V$

- The constraints for the ESS (batteries) are similar to the EVs. The charging and discharging of the ESS can not occur simultaneously. Two binary variables are needed for each ESS to include this constraint in the model (25). 
$X_{(E, t)}+Y_{(E, t)} \leq 1 \quad \forall t, \forall E$

- The energy stored in the ESS by the end of each time period depends on the initial level of energy and the charging and discharging power of the ESS during that time period (26).

$E_{\text {Stored }(E, t)}=E_{\text {Stored }(E, t-1)}+$

$\eta_{c(E)} \times P_{\text {Charge }(E, t)} \times \Delta t-\frac{1}{\eta_{d(E)}} \times P_{\text {Discharge }(E, t)} \times \Delta t \quad \forall t, \forall E$

- Discharge limit for each ESS considering battery discharge rate:

$P_{\text {Discharge }(E, t)} \leq P_{\text {Dischargelimit }(E, t)} \cdot X_{(E, t)} \quad \forall t, \forall E$

- Charge limit for each ESS considering battery charge rate:

$P_{\text {Charge }(E, t)} \leq P_{\text {Chargelimit }(E, t)} \cdot Y_{(E, t)} \quad \forall t, \forall E$

- The ESS cannot discharge more than the available energy in the battery (29).

$\frac{1}{\eta_{d(E)}} \cdot P_{\text {Discharge }(E, t)} \cdot \Delta t \leq E_{\operatorname{Stored}(E, t-1)} \quad \forall t, \forall E, \Delta t=1$

- The ESS cannot charge more than the maximum battery capacity (30).

$\eta_{c(E)} \cdot P_{\text {Charge }(E, t)} \cdot \Delta t \leq E_{\text {BatCap }(E)}-E_{\operatorname{Stored}(E, t-1)} \quad \forall t, \forall E, \Delta t=1$

- The capacity limit for each ESS is considered with constraint (31).

$E_{\text {Stored }(E, t)} \leq E_{\text {BatCap }(E)} \quad \forall t, \forall E$

- Minimum stored energy to be guaranteed at the end of period $t$ for each ESS:

$E_{\text {Stored }(S T, t)} \geq E_{\text {MinCharge }(S T, t)} \forall t \in\{1, \ldots, T\} ; \forall S T \in\left\{1, \ldots, N_{S}\right\}$

- Demand reduction of each load due to the DR programs is constrained with (33).

$P_{\text {LoadDR }(L, t)} \leq P_{\text {LoadDRMaxLimit }(L, t)} \quad \forall t, \forall L$

\section{Multi-dimensional signaling method}

A signaling approach is presented in [34,37] to improve the PSO's performance to solve the ERM problems. The applicability of conventional methods is limited to some PSO-derived approaches, which is not suitable for solving Quantum-based PSO, DSA or Genetic-based approaches. The reason is related to its design because the previous method influences the particles' direction by changing the velocities' limit, which is a particular feature 
of most PSO-based approaches but not the case of QPSO which does not rely on the velocity of particles [35]. The previous signaling method is unable to signal an individual particle in the PSO's swarm but instead influencing the whole population with abrupt changes in the velocities. Taking into account the previous limitation, this paper develops a new method to improve population-based metaheuristics, which we call multidimensional signaling.

Non-convex MINLP problems are the most generalized form of global optimization problems, which involve continuous and discrete variables with non-linear equality and inequality constraints. Many real world constrined problems are modeled as MINLP problems [46]. The general form of a MINLP problem can be represented in the following algebraic form [46]:

$$
\begin{array}{ll}
\underset{x, y}{\operatorname{Minimize}} f(x, y) & \\
\text { s.t. } & \\
g_{i}(x, y)=0 & i=1, \ldots, n_{e q} \in \square \\
g_{i}(x, y) \geq 0 & i=n_{e q}+1, \ldots ., n \in \square \mathrm{n} \\
x_{l} \leq x \leq x_{u} & x_{l}, x_{u} \in \square \\
y_{l} \leq y \leq y_{u} & y_{l}, y_{u} \in \square \\
x \in \square^{n_{1}}, y \in \square^{n_{2}}, n_{1} \in \square, n_{2} \in \square
\end{array}
$$

Where $f(x, y)$ is the objective function, the decision variable $x$ is a vector of $n_{1}$ discrete variables and $y$ is a vector of $n_{2}$ continuous variables. The problem has $n_{e q}$ equality constraints and $\left(n-n_{e q}\right)$ inequality constraints. $x_{l}, x_{u}, y_{l}, y_{u}$ are the lower and upper bounds of the discrete and continuous variables respectively [46].

Penalty methods belong to a general approach that can solve MINLP problems, with no continuity, differentiability and convexity requirements. The purpose of these approaches is to obtain the suitable penalty values in such a way that the optimal solutions of the penalty function corresponds to a local optimal solution of (34) [47]. In penalty function approaches, a penalty term is added to the objective function to penalize the infeasible solutions [48]. The objective function when applying the penalty method transforms to form $\underset{x, y}{\operatorname{Minimize}} f(x, y)+p(x, y)$, where $p(x, y)$ is the penalty function on violated constraints [48]. The penalty function for the MINLP problem (34) can be writtem as follows [47]:

$$
P(x, y)=c \cdot\left[\sum_{i=1}^{n_{e q}}\left(g_{i}(x, y)\right)^{\lambda}+\sum_{i=n_{e q}+1}^{n}\left(\max \left(0, \mathrm{~g}_{i}(x, y)\right)\right)^{\lambda}\right]
$$

Where $\lambda$ is a constant no less than 1 , and $c$ is a positive penalty factor which is greater than $c^{*} . c^{*}$ depends on the value of $\lambda$. It can be statistically chosen or dynamically adjusted [47]. 
Fig. 2 shows a flowchart representing the common procedure in population-based metaheuristics and the MD signaling stages. For the purpose of this work the interest relies on the evaluation and evolution stage for the MD signaling. The proposed signaling method operates in both stages as can be seen in the figure. A signaling matrix is used to enable the logic communication between evaluation stage and evolution stage. In stage 1 (evaluation stage), this matrix is changed if conditions match, and later, in stage 2 (evolution stage), the matrix is interpreted and the respective variables mutated or changed. After this, the matrix is reinitialized to zeros, ready for the iteration. The process is described with more detail in the next subsection.

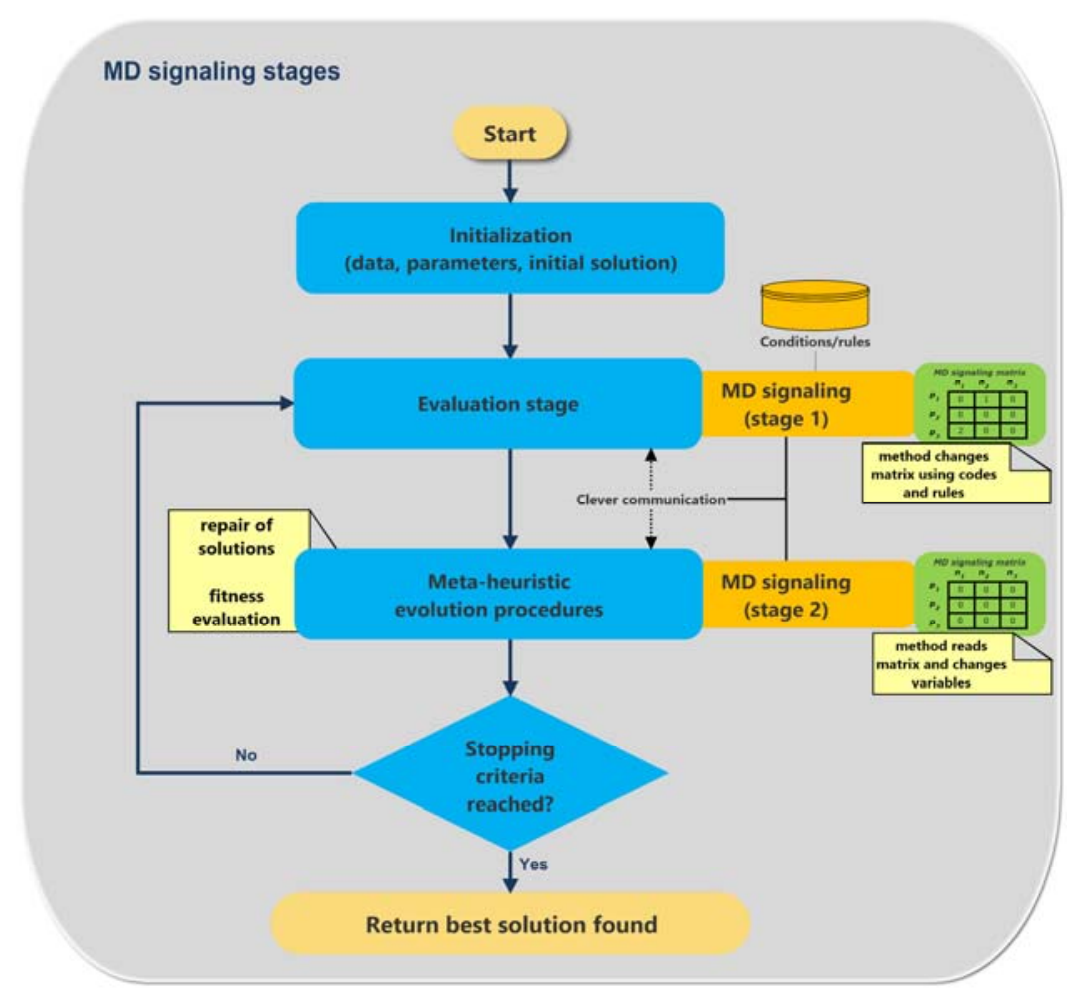

Fig. 2. MD signaling procedure in metaheuristics

\subsection{Formal definition}

The multi-dimensional signaling is described with more detail in this subsection. The method relies on exchanging signals between the core process of the metaheuristic, i.e. movement or evolution, and the identification of violations and evaluation of the solutions. The signaling is important in both moments, namely identifying critical variables in the evaluation stage that should be treated differently in the evolution stage.

For the signaling mechanism we define the signaling matrix $S^{p \times n} \in \square^{p \times n}$ as a matrix with $p$ elements in the population-based algorithm (population size, e.g. number of particles in PSO) and $n$ the number of decision 
variables of the optimization problem of (35). $S$ is reinitialized with zeros at each iteration $k$ before the evaluation stage. The signal function (36) changes the values of $S$ in each $k$ iteration at evaluation stage if a violation of a given constraint is found or if a custom condition $\vartheta$ is true.

$$
S= \begin{cases}\operatorname{sigFun}_{z}(S), & \text { if } g\left(\mathrm{c}_{z}\left(x^{e}\right)\right) \geq 0, z \in I, e \in p \\ \operatorname{sigFun}_{i}(\mathrm{~S}), & \text { if } \vartheta_{i}=\text { true }, i \in \square, i \neq z \\ 0, & \text { otherwise }\end{cases}
$$

where $z$ corresponds to the list of $m$ constraints that are suitable for signaling and $\vartheta_{i}$ to the $i$ condition.

The engineer and/or programmer should identify which variables and conditions are best suitable to be signaled during the evaluation stage. The algorithm should be designed to evaluate the conditions and identify which variables should be signaled across iterations to improve solution fitness and/or constraint handling in the best way. The criteria to define which variables are chosen to be signaled should include the following:

- variables that can easily relieve constraint violations if changed in a certain direction;

- variables that cannot be changed by direct repair method;

- variables that can improve fitness function.

\subsection{Toy example}

An example is given to better understand the method. Let us consider the energy scheduling problem to minimize the operation costs. The problem includes several DGs and one ESS. Consider that a condition $\vartheta$ exists such that $\operatorname{sigFun}_{1}(S)$ also exists. Condition $\vartheta_{1}$ is defined as true if the system's marginal price is lower than the ESS charging price. For each condition $\vartheta_{1}$ a signal code is assigned, which is stored in the signaling matrix if the condition is verified. Assume that the defined signal for $\vartheta_{l}$ is 1 and that this signal means that the variable signaled should change its value towards its upper bound. In the evaluation stage $S$ is changed whenever each condition is true or $g\left(\mathrm{c}_{z}(x)\right) \geq 0$. The pseudo-code bellow illustrates the procedure of $\operatorname{sigFun}_{1}(S)$ at evaluation stage:

1. start

2. if $\vartheta_{1}$ is true // the condition $\vartheta$ checks if system's marginal price is lower than ESS charging price

3. then // if the condition is true execute the following actions

4. $\quad v=$ get set of particles to signal (e.g. randomly, all or one particle)

5. $\quad u=$ get the indices in $x^{e}$ that correspond to the ESS variable(s) 


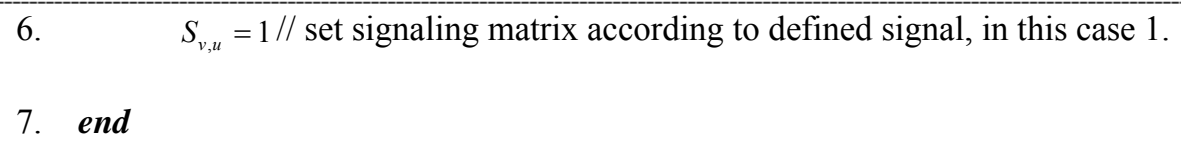

At the evolution stage, before executing the typical movement in meta-heuristic, the $S$ matrix is read. For the previous case the ESS variable will jump to the upper limit as the signal code meant that. A random number can be multiplied by the upper limit of the variable to allow more diversity. It can also be desirable that there is no effect of the signaling process by using a probability of executing the signal change. The above example shows how to setup a condition that could improve fitness function by charging ESS when system price is low, improving profit. Most metaheuristics might be able to reach the optimal point but it will take much more iterations to converge or simply fail to find it as a result of being trap in a local optima.

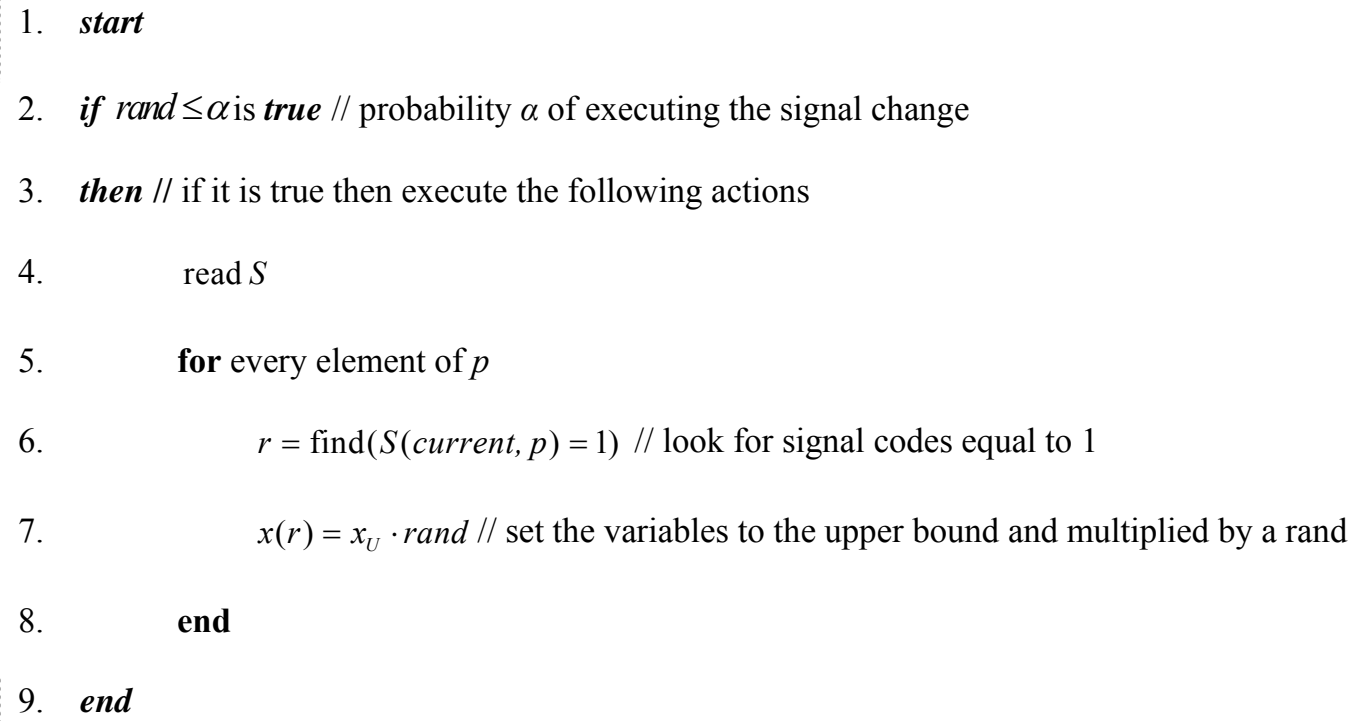

\section{Metaheuristics-based methods}

This section presents a brief review of the methods implemented in the ERM problems. It is essential to develop efficient decision support methods to solve energy resource scheduling, because the operators need to test several scenarios, which makes time requirements crucial for this applications $[13,34,49]$. Deterministic optimization techniques require increasing computational resources to deal with the large-scale energy scheduling problem [6]. Artificial intelligence department has demonstrated to have good alternatives to conventional optimization in order to solve complex and real problems that present non-convexity, nondifferentiability and discontinuity. For example, Genetic Algorithms (GA), Particle Swarm Optimization (PSO) 
and other evolutionary algorithms have been used to solve complex problems. PSO has less tweaking parameters than GA, i.e. GA requires more tuning regarding operators for crossover, mutation and selection of the best solutions. Moreover, the overhead resulting from implementing these operators in GA makes this technique less practical than algorithms comparable to PSO [50,51]. Recently, combining methods based on conventional and/or evolutionary optimization seems to be a promising path, especially the premature convergence and stagnation, which can compromise the solutions quality of the metaheuristics $[52,53]$.

\subsection{Particle Swarm Optimization}

A PSO-based approach is used with some modifications from the traditional one [54]. The strategic parameters inertia, memory, and cooperation, introduced in [55], are used in this PSO implementation as part of the mutation process. The mutation of the strategic parameters is applied directly to the original swarm rather than the replicated swarm as in [55]. Adding mutation of the strategic parameters, as in [55], demonstrated to improve the results of the original PSO. Replication requires at least twice the amount of particles and the number of evaluations. Therefore, the replication and selection of the particles, implemented in [55], was not considered in this version to reduce computation time. In the case of the PSO implemented in this paper, equations (37) and (38) represent the maximum and minimum velocities of the particles. The velocity clamping factor $\left(C_{\text {factor }}\right)$ is a parameter that linearly influences the velocity limits. This parameter should be between 0 and 1 . The minimum velocity is the negative of the maximum velocity limits calculated using the velocity clamping factor. More details about the PSO implemented by the authors can be found in [34]. Also, further reading about PSO can be found in $[38,50]$

$$
\begin{aligned}
& v_{\text {max }}=C_{\text {factor }} *\left(x_{\text {max }}-x_{\text {min }}\right) / 2 \\
& v_{\text {min }}=-v_{\text {max }}
\end{aligned}
$$

where:

$\begin{array}{ll}v_{\max } & \text { Maximum velocity limit of variables } \\ v_{\min } & \text { Minimum velocity limit of variables } \\ x_{\max } & \text { Upper bounds of variables } \\ x_{\min } & \text { Lower bounds of variables } \\ C_{\text {factor }} & \text { Velocity clamping factor }\end{array}$




\subsection{Quantum PSO}

The traditional PSO relies on the convergence to the global best (best solution of the swarm) particle, independently of the position of other particles. This particular feature is the major shortcoming of the method when there are few particles far away from the global best position. QPSO is a new version of PSO, motivated from quantum mechanics [35]. The iterative equation of QPSO is very different from traditional PSO, without velocity vectors, fewer parameters and easier to implement. In order to enhance the global search ability of the particle a mean best position is introduced. In QPSO the lagged particles are not abandoned by the swarm because they affect the mean best position, therefore the lagged particles are shifted towards the rest of the swarm. The mean best position is intrinsically related with the movement equation of QPSO (41). The particles' distribution affects the convergence rate; however, QPSO can provide stronger global search ability than traditional PSO [35].

Equation (39) presents the local focus equation which is a random point located within the hyperplane constructed between $P_{i, n}$ and $G_{n}$ in the search space. The local focus is then used in equation (40). The contraction-expansion (CE) coefficient $\alpha$, which is vital to the dynamical behavior of an individual particle and the convergence of the algorithm. The algorithm decides to add or subtract the second term of equation (40) using a probability of 50\%. A comprehensive reading concerning QPSO can be found in $[35,56,57]$.

$$
\begin{aligned}
& p_{i, n+1}^{j}=\varphi_{i, n}^{j} P_{i, n}^{j}+\left(1-\varphi_{i, n}^{j}\right) G_{n}^{j} \\
& X_{i, n+1}^{j}=p_{i, n}^{j} \pm \alpha\left|X_{i, n}^{j}-C_{n}^{j}\right| \ln \left(\frac{1}{u_{i, n+1}^{j}}\right) \\
& \text { where: } \\
& C_{n}^{j} \quad \text { Mean best position which can be defined by the average of the personal best positions of all } \\
& \text { particles } \\
& G_{n}^{j} \quad \text { The global best of particle at the } n \text {th iteration } \\
& P_{i, n}^{j} \quad \text { The } j \text { th component of the personal best of particle } i \text { at the } n \text {th iteration } \\
& p_{i, n}^{j} \quad \text { The } j \text { th component of the local focus } p_{i, n} \text { of particle } i \text { at the } n \text {th iteration } \\
& u_{i, n+1}^{j} \quad \text { Random uniformly distributed sequence }[0,1] \\
& X_{i, n}^{j} \quad \text { Position of the particle } i \text { th at the } n \text {th iteration } \\
& \varphi_{i, n}^{j} \quad \text { Random uniformly distributed sequence [0,1]. }
\end{aligned}
$$

\subsection{Differential search algorithm}


There are several computational intelligence algorithms representing the behavior of nature. Some of the most popular algorithms in this category include Particle Swarm Optimization (PSO), Cuckoo Search (CS), Firefly Algorithm (FA), Artificial Bee Colony (ABC) and Ant Colony optimization (ACO). The Differential Search Algorithm (DSA) is a new metaheuristic that demonstrated to be more effective in several benchmark problems [58]. Like the previous ones, the DSA algorithm is inspired in the nature, in this case in the migration of living beings. The migrating species of living beings constitutes a population with a large number of individuals, which can be described by a Bownian-like random-walk model [59]. This behavior can be computational modeled to solve optimization problems. Therefore, the population in DSA corresponds to a superorganism migrating to a global optimum solution of the problem. In the course of this migration, the superorganism tests whether some randomly selected positions are suitable for temporarily basis. If the tested position is suitable, the members of the superorganism that made this discovery immediately settle at the discovered position. A flowchart representing the DSA procedure can be seen in [58].

A member of a superorganism, in its initial position, is generated between the upper and lower bounds of the problem variables. Moroever, The DSA has only two control parameters, $p_{1}$ and $p_{2}$ [33] which provide a trialpattern generation strategy for morphogenesis in order to determine the best solutions for the optimization problem. For the direction generation strategy (donor), DSA has four methods for controlling the superorganism evolution as referred in [58]. This evolution is controlled by the generation of the donor. The bijective method evolves the superorganism in random directions, the subjective evolves toward some of the best solutions, the elitist-random directs to one of the best while the elitist method evolves the superorganism toward to "the best" solution.

The expression for a stopover site position is given by the equation (41).

$$
\text { Stopover }=\text { Superorganism }+R \times(\text { donor }- \text { Superorganism })
$$

The scale factor $R$ in equation (42) can be defined by a random number using a gamma distribution with a shape parameter in $A$ and scale parameter in $B$ as is shown by equation (42), simulating a pseudo-stable walk movement. Actually, the generated scale factor $R(42)$ is fundamental to control the magnitude of the change in positions of the members of the superorganism (41). Further reading can be found in $[33,36]$.

$$
R=\frac{1}{\Gamma(A, B)}
$$




\subsection{Two-stage hybrid deterministic-metaheuristic}

A relaxed MILP formulation is implemented in this paper using TOMLAB. The MILP can afford efficient large-scale solutions but using a relaxation approach may results in using solutions that cause network problems under certain conditions. Hence, a combination between DSA and MILP is proposed which can provide higher solution quality while validating the $\mathrm{AC}$ network equations using a power flow. One individual of the population in DSA is replaced by the solution of MILP. The remaining population remains unchanged, i.e. randomly generated.

\section{Implementation}

In this section information about the heuristics implementation and MD signaling is provided.

\subsection{Metaheuristics implementation}

The decision variables of the optimization problem under study are coded into vector space. DG variables are between the limits of the respective generators for active and reactive power. The ESS and V2G variables are positive if charging and negative otherwise. The DR variables are coded in metaheuristics between the maximum and minimum possible DR use (see 3.3). The binary variables are implemented as continuous but using a rounding mechanism, i.e. a continuous variable between 0 and 1 is rounded to 0 or 1 depending on its value. To improve the metaheuristics' efficiency and effectiveness, a heuristic mechanism is implemented to pre-dispatch the cheapest DG resources. The DGs' units generation and commitment are sorted by merit order according to their costs, except from renewable generation which is not dispatchable. The heuristic pre-schedules the DGs units, balancing the generation and the demand in each period, taken into account the metaheuristics randomly seek for EVs, ESS and DR variables. This variables do not join any pre-schedule heuristic. It is important to note that only half of the members of the population/swarm are chose (randomly) to join this process, whereas the remaining are not changed by this heuristic. After this process, a power flow is run before the final evaluation (in each iteration) by the metaheuristics process. Moreover, this process is common for all the implemented metaheuristics in the paper to enable a fair comparison when testing the MD signaling method effect. The power flow used to calculate network parameters for each solution obtained is implemented as in [60]. This allows the metaheuristics to have information to evaluate the network constraints, namely lines thermal limits, voltage magnitude and angle boundaries. The violations identified are handled by using penalties in the fitness function, signaling mechanism and using direct repair, i.e. correcting a solution by changing the variable value, whenever 
possible, and re-evaluating the objective function without adding further penalties. This implies knowing the problem structure very well, e.g. if some storage charging value causes higher amount than the ESS's capacity in a given period, this variable can be directly reduced without causing violation in the capacity. In this work the standard metaheuristics have all the implemented features mentioned in this subsection except from the signaling mechanism, which is only activated to demonstrate its effectiveness and allow comparison.

\subsection{Multi-dimensional signaling implementation}

To implement the multi-dimensional signaling for the ERM problem three signaling codes are defined, namely code $-1,0$ and 1 . Code -1 means that the variables signaled with this code will decrease its value close to their lower bounds, i.e. the lower bound of the variable multiplied by a rand value with uniform distribution. Code 0 implies that the variables change to 0 . Finally code 1 is assigned to increase variables' value close to the upper bounds. Taking into account these defined codes, the conditions implemented for the ERM problem are:

\section{Technical conditions:}

- Signal EVs and ESS to charge (code 1) and if available decrease local reactive generation (code -1) when voltages violations (upper limit) are found nearby;

- Signal EVs and ESS to discharge (code -1) and if available increase local reactive generation (code -1) when voltages violations (lower limit) are found nearby;

- Signal consumption to decrease (incl. selling energy) if demand is higher than the available generation;

Fitness improvement conditions:

- Signal V2G/ESS to discharge if the system marginal cost is higher than the contracted price (code -1);

- Signal V2G/ESS to charge if the system marginal cost is lower than the contracted price (code 1);

- Signal DR to use if the system marginal cost is higher than the DR contracted price plus the load income (code 1);

- Signal DR with code 0 if the system marginal cost is lower than the contracted price plus the load income;

- Signal selling energy (sell to market) if the system marginal cost is lower than the selling price (code 1);

- Signal selling energy with code 0 if the system marginal cost is higher than the selling energy price.

\section{Case study}

Tests were conducted in two network systems, namely a 33-bus $12.66 \mathrm{kV}$ distribution test network adapted from [61] and a 180-bus $30 \mathrm{kV}$ real distribution network from Portugal. The 33-bus test system is a $12.66 \mathrm{kV}$ 
network with one substation, $32 \mathrm{MV}$ load points, $1800 \mathrm{EVs}, 67$ DGs including a large wind unit, 10 external suppliers and 15 ESSs. The 180-bus test system is a real Portuguese distribution $30 \mathrm{kV}$ network with one substation adapted to a future scenario, $90 \mathrm{MV}$ load points, $6000 \mathrm{EVs}, 116 \mathrm{DG}$ units, 1 external supplier, and 7 ESSs. The external suppliers are modeled in bus 1 corresponding to the substation location and connection with the main grid. In both test cases Demand Response (DR) with Direct Load Control (DLC) was considered. The benefit for DLC contracts is set to $0.02 \mathrm{~m} . \mathrm{u} . / \mathrm{kWh}$ in both cases. The consumers receive this benefit per each unit of energy reduced, instead of paying the VPP the contracted supply price, which is $0.14 \mathrm{~m} . \mathrm{u} . / \mathrm{kWh}$ for the 33 bus test system and 0.16 m.u./kWh for the 180-bus test system. The selling energy price, i.e. energy sold to external, is considered $0.14 \mathrm{~m} . \mathrm{u} . / \mathrm{kWh}$ in both cases. The considered prices and the DG capacity of the test systems have taken into account the observations made in the vision of [62]. The scenarios of EVs for both systems were developed using the tool presented in [63]. The details of each test system are described in the following subsections ${ }^{1}$. The work was developed in MATLAB R2014a 64 bits, and TOMLAB 64 bits software [39] using a computer with one Intel Xeon E5-1650 processor and 6 GB of RAM running Windows 8.1.

\subsection{3-bus network scenario}

For the 33-bus test system a fleet of 1800 EVs with V2G capabilities is considered with total energy demand predicted for the trips is equal to $13.77 \mathrm{MWh}$ corresponding to an average of $7.65 \mathrm{kWh}$ per vehicle and a total of 2553 trips (average of 1.4 trips per vehicle). The discharging cost considered for the EVs and ESS is set to 0.19 m.u./kWh in this scenario. A charging/discharging efficiency of $77 \%$ is considered for EVs and $90 \%$ for ESS. The total capacity from the external suppliers is $6.20 \mathrm{MW}$ in the considered time horizon. Table 1 presents the considered costs and the capacity of the system. The total forecasted regular load without EVs, ESS and DR is $102.97 \mathrm{MWh}$.

Table 1. 33-bus grid scenario characterization

\begin{tabular}{|c|c|c|c|c|c|}
\hline Resources & $\begin{array}{l}\text { Min. price } \\
\text { (m.u./kWh) }\end{array}$ & $\begin{array}{l}\text { Max. price } \\
\text { (m.u./kWh) }\end{array}$ & $\begin{array}{c}\text { Min. capacity } \\
(M W)\end{array}$ & $\begin{array}{c}\text { Max. capacity } \\
(M W)\end{array}$ & $\begin{array}{c}\text { Units } \\
\#\end{array}$ \\
\hline Biomass & 0.09 & 0.09 & 0.38 & 0.38 & 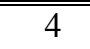 \\
\hline $\mathrm{CHP}$ & 0.06 & 0.06 & 1.15 & 1.15 & 15 \\
\hline Fuel Cell & 0.15 & 0.15 & 0.11 & 0.11 & 7 \\
\hline Small Hydro & 0.07 & 0.07 & 0.07 & 0.07 & 2 \\
\hline
\end{tabular}

\footnotetext{
${ }^{1}$ The complete data for the test systems can be found in http://www.gecad.isep.ipp.pt/ies/public-data/swevo/
} 


\begin{tabular}{l|c|c|c|c|c}
\hline \hline \multicolumn{1}{c|}{ Resources } & $\begin{array}{c}\text { Min. price } \\
(\mathbf{m . u .} / \mathbf{k W h})\end{array}$ & $\begin{array}{c}\text { Max. price } \\
(\mathbf{m . u .} / \mathbf{k} \boldsymbol{W h})\end{array}$ & $\begin{array}{c}\text { Min. capacity } \\
(\boldsymbol{M} \boldsymbol{W})\end{array}$ & $\begin{array}{c}\text { Max. capacity } \\
(\boldsymbol{M} \boldsymbol{W})\end{array}$ & $\begin{array}{c}\text { Units } \\
\#\end{array}$ \\
\hline \hline PV & 0.20 & 0.20 & 0.00 & 0.84 & 31 \\
\hline $\begin{array}{l}\text { Waste-to- } \\
\text { energy }\end{array}$ & 0.10 & 0.10 & 0.01 & 0.01 & 1 \\
\hline Wind & 0.15 & 0.15 & 0.18 & 0.89 & 6 \\
\hline Large Wind & 0.07 & 0.07 & 1.58 & 1.80 & 1 \\
\hline $\begin{array}{l}\text { External } \\
\text { Suppliers }\end{array}$ & 0.09 & 0.30 & 6.20 & 6.20 & 10 \\
\hline V2G & 0.19 & 0.19 & 0.00 & 5.72 & 1800 \\
\hline ESS & 0.19 & 0.19 & 0.90 & 0.90 & 15 \\
\hline DR & 0.02 & 0.02 & 0.60 & 1.17 & 32 \\
\hline \hline
\end{tabular}

To evaluate the developed methodology under the 33-bus scenario an extensive setup was implemented using MINLP (GAMS), MILP (TOMLAB), and the metaheuristics PSO, QPSO and DSA. The metaheuristics were tested using the multi-dimensional (MD) signaling approach and compared with the standard version without signaling. The parameters used for the metaheuristics can be seen in Table 2. These parameters were obtained by empirical testing and previous studies available on the literature [33].

Table 2. Metaheuristics-based approaches parameters

\begin{tabular}{|c|c|c|c|c|}
\hline Parameters & PSO & QPSO & DSA & HDSA \\
\hline \#Individuals & \multicolumn{4}{|c|}{10} \\
\hline Initial Solution & \multicolumn{3}{|c|}{ Random } & $\begin{array}{l}\text { Random and } \\
\text { MILP }\end{array}$ \\
\hline Inertia Weight & \multirow{4}{*}{$\begin{array}{c}\text { Gaussian } \\
\text { mutation } \\
\text { weights between } \\
0 \text { and } 1 \\
\text { (initial weights } \\
\text { randomly } \\
\text { generated) }\end{array}$} & & & \\
\hline $\begin{array}{l}\text { Acceleration Coefficient Best } \\
\text { Position }\end{array}$ & & & & \\
\hline Cooperation Coefficient & & & & \\
\hline Perturbation Coefficient & & & & \\
\hline Mutation learning parameter $(\delta)$ & 0.20 & & & \\
\hline Max. Velocities & \multirow{2}{*}{$\begin{array}{c}\text { Velocity } \\
\text { clamping factor } \\
\text { equation }\end{array}$} & & & \\
\hline Min. Velocities & & & & \\
\hline Velocity Clamping Factor $\left(C_{\text {factor }}\right)$ & 1 & & & \\
\hline Alpha $(\alpha)$ & & 0.3 & & \\
\hline Min. iterations & \multicolumn{4}{|c|}{500} \\
\hline
\end{tabular}




\begin{tabular}{|c|c|c|c|c|c|}
\hline \multicolumn{2}{|c|}{ Parameters } & PSO & QPSO & DSA & "HDSA \\
\hline \multirow{3}{*}{$\begin{array}{c}\text { Stopping } \\
\text { Criteria }\end{array}$} & Max. iterations & \multicolumn{4}{|c|}{2000} \\
\hline & Threshold & \multicolumn{4}{|c|}{$10^{-9}$} \\
\hline & Threshold iterations & \multicolumn{4}{|c|}{400} \\
\hline \multicolumn{2}{|c|}{ Scale-factor } & & & \multicolumn{2}{|c|}{ Pseudo-stable walk } \\
\hline \multicolumn{2}{|c|}{ Morphogenesis $p_{1}$ and $p_{2}$} & & & \multicolumn{2}{|c|}{ Random between 0 and 0.3} \\
\hline \multicolumn{2}{|c|}{ Direction method } & & & \multicolumn{2}{|c|}{ Elitist } \\
\hline \multicolumn{2}{|c|}{ Max. Positions } & \multicolumn{4}{|c|}{ Equal to the upper bounds of the variables } \\
\hline \multicolumn{2}{|c|}{ Min. Positions } & \multicolumn{4}{|c|}{ Equal to the lower bounds of the variables } \\
\hline
\end{tabular}

Table 3 reveals the benchmarks made using the aforementioned methods over a large number of runs, i.e. 100

in this case. For each metaheuristic a configuration with the MD signaling was tested (cf. column MD signaling in Table 3). Furthermore, for QPSO a different parameter configuration was tested, namely with alpha linearly decreasing from 1.0 to 0.3 (global to local exploration) and with alpha fixed to 0.3 (local exploration). Regarding DSA metaheuristic different directions methods were tested, namely bijective and elitist direction method. The bold results indicate the best configuration obtained within each method.

The percentage of feasible solutions obtained with MD signaling activated was 100\% in PSO, QPSO and DSA methods while without MD signaling activated it varied significantly, i.e., $60 \%$ in standard PSO, 0\% in QPSO with fixed alpha, 100\% in QPSO with linearly decreasing alpha, 15\% in DSA elitist and 85\% in DSA bijective. HDSA and MILP obtained feasible solutions in this scenario. The violations verified were mainly due to voltages issues in the network. To evaluate the robustness of the methods the standard deviation was measured, this value varied significantly between the presented configurations. The standard deviation was below 11 m.u. for HDSA, PSO, QPSO (fixed alpha) and DSA with MD signaling. The same measure was as high as 58 m.u. in standard PSO without MD signaling. In QPSO (decreasing alpha) with MD signaling the standard deviation was $81 \mathrm{~m} . \mathrm{u}$. due to the presence of one outlier, while, without the outlier the standard deviation would be about 10 m.u. Fig. 3 shows the box plot for the average results achieved in the performed tests, namely minimum, median and $75^{\text {th }}$ (Q3) and $25^{\text {th }}$ (Q1) percentile and maximum profits for each metaheuristic. Standard QPSO without MD signaling is not included in this figure because all the solutions returned were infeasible.

The MILP method did not consider the full AC network equations as in metaheuristics and MINLP, hence MILP obtained higher profits as power losses were not considered. However, after evaluating the MILP results 
with the full AC power flow used in metaheuristics, the profit was corrected to 5478 m.u. The MILP method can be unrealistic but was compared for reference in this paper. The MINLP considered the network equations but took an excessive 14 hours to solve the optimization problem while the metaheuristics took, on average, about 6 minutes and HDSA only 163 seconds (30 seconds with 100 iterations).

To evaluate the impact on computing system resources a memory test was made using MATLAB memory profiler tool for the 33-bus scenario. This method reported the peak memory for each function. The metaheuristics presented an average peak memory use of 4 MB, MILP using TOMLAB reported 52 MB of use and HDSA peaked 53 MB. GAMS memory used was measured by analyzing windows memory information which was around $270 \mathrm{MB}$.

Table 3. 33-bus scenario methods' results (averaged over 100 runs)

\begin{tabular}{|c|c|c|c|c|c|c|c|}
\hline Method & $\begin{array}{c}\text { Changed } \\
\text { parameters }\end{array}$ & $\begin{array}{c}\text { MD } \\
\text { signaling }\end{array}$ & $\begin{array}{c}\text { Income } \\
\text { (m.u.) }\end{array}$ & $\begin{array}{c}\text { Operation } \\
\text { Costs (m.u.) }\end{array}$ & $\begin{array}{c}\text { Profits } \\
\text { (m.u.) }\end{array}$ & $\begin{array}{c}\text { Execution } \\
\text { time (s) }\end{array}$ & \#Iterations \\
\hline \multirow{2}{*}{ PSO } & & $\overline{Y Y}$ & 16,995 & 11,428 & $\overline{5527}$ & 331 & 1388 \\
\hline & & $\mathrm{N}$ & 17,960 & 12,942 & 5018 & 477 & 1988 \\
\hline \multirow{4}{*}{ QPSO } & & $\mathbf{Y}$ & 16,944 & 11,400 & 5543 & 377 & 1495 \\
\hline & $\alpha(1 \rightarrow 0.3)^{2}$ & $\mathrm{Y}$ & 16,988 & 11,493 & 5495 & 498 & 1930 \\
\hline & & $\mathrm{N}$ & \multicolumn{5}{|c|}{$100 \%$ infeasible solutions } \\
\hline & $\alpha(1 \rightarrow 0.3)^{2}$ & $\mathrm{~N}$ & 21,103 & 17,114 & 3989 & 472 & 1924 \\
\hline \multirow{4}{*}{ DSA } & & $\mathbf{Y}$ & 17,220 & 11,658 & 5562 & 328 & 1790 \\
\hline & Bijective & $\mathrm{Y}$ & 17,088 & 11,576 & 5512 & 355 & 1976 \\
\hline & & $\mathrm{N}$ & 20,405 & 16,243 & 4162 & 399 & 2000 \\
\hline & Bijective & $\mathrm{N}$ & 19,603 & 15,105 & 4498 & 413 & 2000 \\
\hline \multirow{2}{*}{ HDSA } & & $\mathbf{Y}$ & 17,215 & 11,630 & 5586 & 163 & 959 \\
\hline & Max. 100 it. & $\bar{Y}$ & 17,221 & 11,637 & 5584 & 30 & 100 \\
\hline MILP & & & 17,110 & 11,256 & 5854 & 10 & \\
\hline MINLP & & & 16,968 & 11,301 & 5667 & $\begin{array}{l}50,058 \\
\end{array}$ & \\
\hline
\end{tabular}




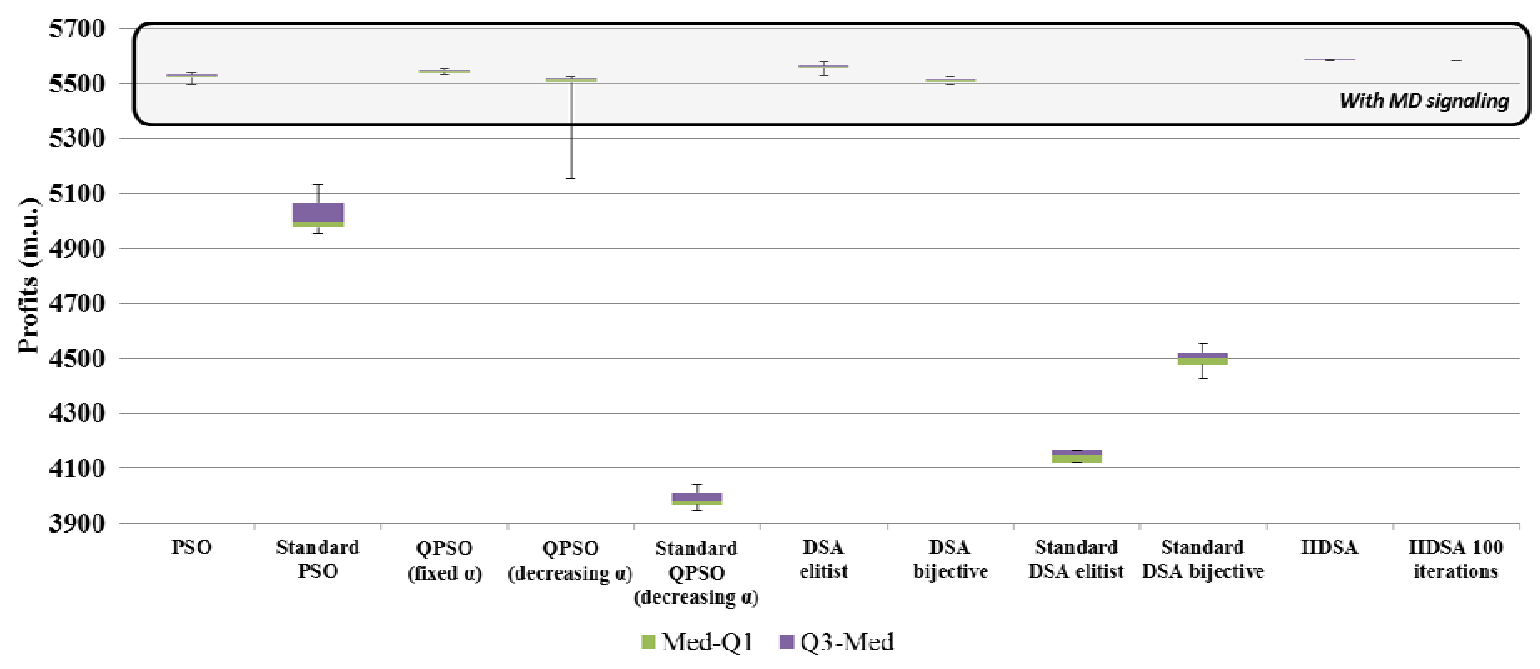

Fig. 3. Box plot for the average results of 100 runs in the 33-bus scenario.

Fig. 4 shows the average fitness values over 100 runs representing the convergence test with iterations fixed to 2000 for comparison purposes. It is worth noting that a negative fitness value represents a positive profit because the algorithm was setup to minimize the objective function (3). The configurations that obtained $100 \%$ feasible solutions in all run tests were considered in this plot, namely PSO, QPSO, and DSA with MD signaling, and standard QPSO without MD signaling with linearly decreasing alpha. HDSA was not included in this test because it included a deterministic startup solution.

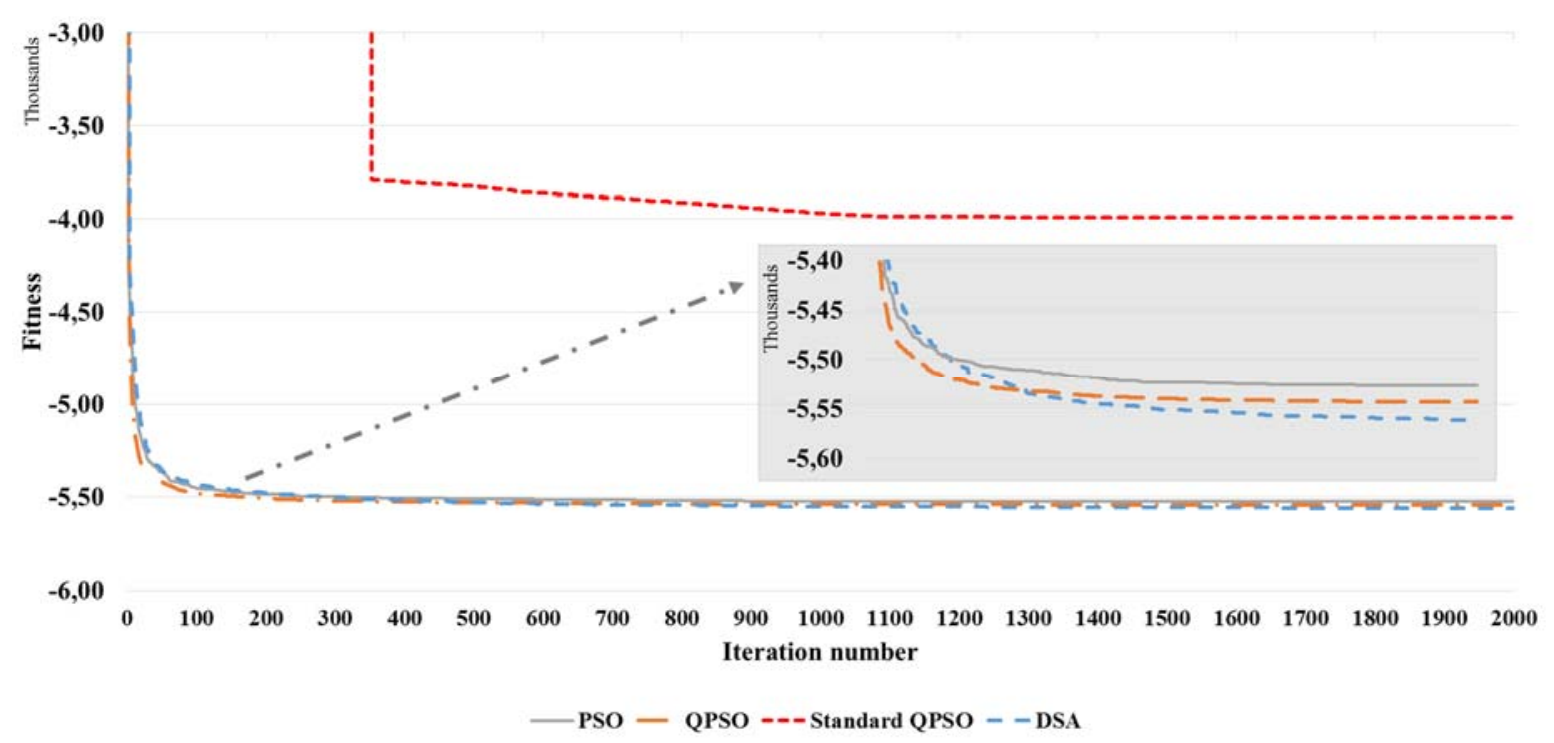

Fig. 4. Convergence test over 100 runs for selected metaheuristics: PSO, QPSO, standard QPSO and DSA. 
The metaheuristics with MD signaling presented very similar convergence properties presenting a fast evolution in the first few iterations and slower improvement onwards. DSA obtained slightly higher fitness on average when compared with QPSO and PSO. QPSO was slightly better than PSO but worse than DSA. Nevertheless, the difference seen in the fitness values of these metaheuristics is somewhat negligible when MD signaling is activated. Without MD signaling only the standard QPSO with linearly decreasing alpha setup was able to find $100 \%$ feasible solutions in all run tests. However, the difficulties of standard QPSO are proven by the convergence curve as it takes more than 300 iterations to make the jump to the $3 \mathrm{k}$ level and then slowly improving up to the $4 \mathrm{k}$ level without never achieving the $5 \mathrm{k}$ level. Standard PSO and DSA are not plotted in the convergence figure because they did not obtained $100 \%$ feasible solutions in all run tests. The average fitness value of standard PSO was higher than standard DSA and standard QPSO but most of them presented violations and for this reason the comparison is not really possible (see Table 3).

A direct comparison between two mean best fitness may be misguiding because of the stochastic nature of the algorithms. Therefore, one-way analysis of variance, commonly known as ANOVA, was used to validate the statistical results obtained. The significance level chosen was 0.01 which corresponds to a confidence level of $99 \%$. The null hypothesis is that the means are equal, the alternative hypothesis is that the means are different.

Table 4 shows the results of the ANOVA tests, which consisted in an individual ANOVA test for each comparison, namely DSA with other metaheuristics and HDSA with DSA. The tests indicate that the results obtained previously are significant and valid at the 0.01 significance level. Hence, the null hypothesis is rejected and the alternative hypothesis is accepted, i.e. the means are significantly different. Although, the differences between DSA, PSO and QPSO with MD signaling are statistically different at the 0.01 level, perhaps from the operator standpoint are somewhat negligible, i.e. lower than $0.7 \%$.

Table 4. ANOVA test between the algorithms in the 33-bus scenario

\begin{tabular}{ccccc}
\hline $\begin{array}{c}\text { Compared } \\
\text { algorithm }\end{array}$ & $\begin{array}{c}\text { DSA versus } \\
\text { PSO }\end{array}$ & $\begin{array}{c}\text { DSA versus } \\
\text { QPSO (fixed } \alpha)\end{array}$ & $\begin{array}{c}\text { DSA versus } \\
\text { standard QPSO }\end{array}$ & $\begin{array}{c}\text { HDSA versus } \\
\text { DSA }\end{array}$ \\
\hline$\mu_{1}$ & 5527 & 5562 & & 5586 \\
$\mu_{2}$ & & 5543 & 3989 & 5562 \\
$\sigma_{1}$ & 11 & 7 & 27 & 1 \\
$\sigma_{2}$ & 103 & 44 & 59638 & 11 \\
F-value & $2.39^{-12}$ & $7.93^{-8}$ & Infinitesimal & 92 \\
$p$-value & Significant & Significant & Extremely significant & Significant \\
Significance & & & & \\
\hline \hline
\end{tabular}


Fig. 5 and Fig. 6 present the load and generation scheduling, respectively, as optimized by MINLP in GAMS, MILP in TOMLAB, DSA with MD signaling and HDSA. For the metaheuristics-based approaches a random run was made to obtain the presented results ${ }^{2}$. It can be seen that MINLP approach scheduled more market selling energy (9.03 MWh) than MILP (8.58 MWh), DSA (1.93 MWh) and HDSA (8.40 MWh), The results of MINLP were more dispersed along the day while the others methods were more concentrated in the earlier periods in what concerns the selling energy. The total DG scheduled was very similar between the presented approaches, namely 93.70 MWh, 93.41 MWh, 94.13 MWh and 93.93 MWh in MINLP, MILP, DSA, HDSA, respectively. The total external supplier scheduled was higher in HDSA (30.30 MWh), followed by DSA (29.02 $\mathrm{MWh}$ ) and lower in GAMS and MILP (28.80 MWh). The total DR schedule was higher in DSA (1.24 MWh), followed by MINLP (0.26 MWh) and lower in HDSA and MILP (0.20 MWh). The DR reduce schedule was prominently more intensive in DSA and used in more periods than other approaches as can be seen in the figure. The discharge of EVs and storage was not scheduled by any of the algorithms. The total vehicle charge schedule was higher in DSA (16.04 MWh), followed by HDSA (11.66 MWh), MILP (10.87 MWh) and lower MINLP (9.46 MWh). While MINLP, MILP and HDSA choose to sell more energy to the main grid (market), DSA choose to charge more EVs and charge ESS (1.87 MWh) instead. The total active power losses were lower in MINLP (1.30 MWh), followed by HDSA (1.41 MWh), and higher in DSA (1.58 MWh) while not considered in the model of MILP.

\footnotetext{
${ }^{2}$ The complete results of the 33-bus scenario can be found in http://www.gecad.isep.ipp.pt/ies/public-data/swevo/
} 

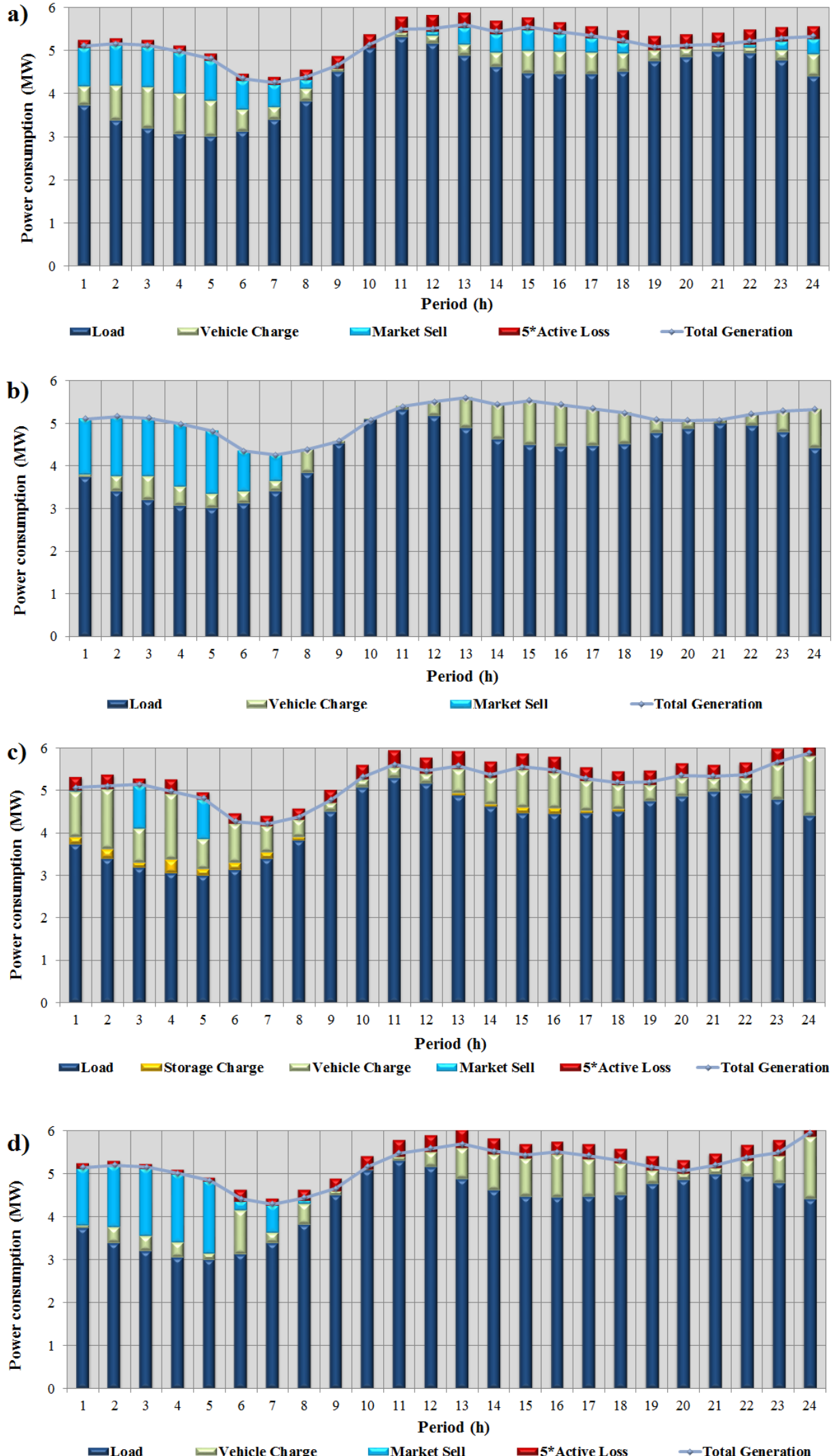

Fig. 5. Load scheduling: a) MINLP b) MILP c) DSA d) HDSA (top-to-bottom). 

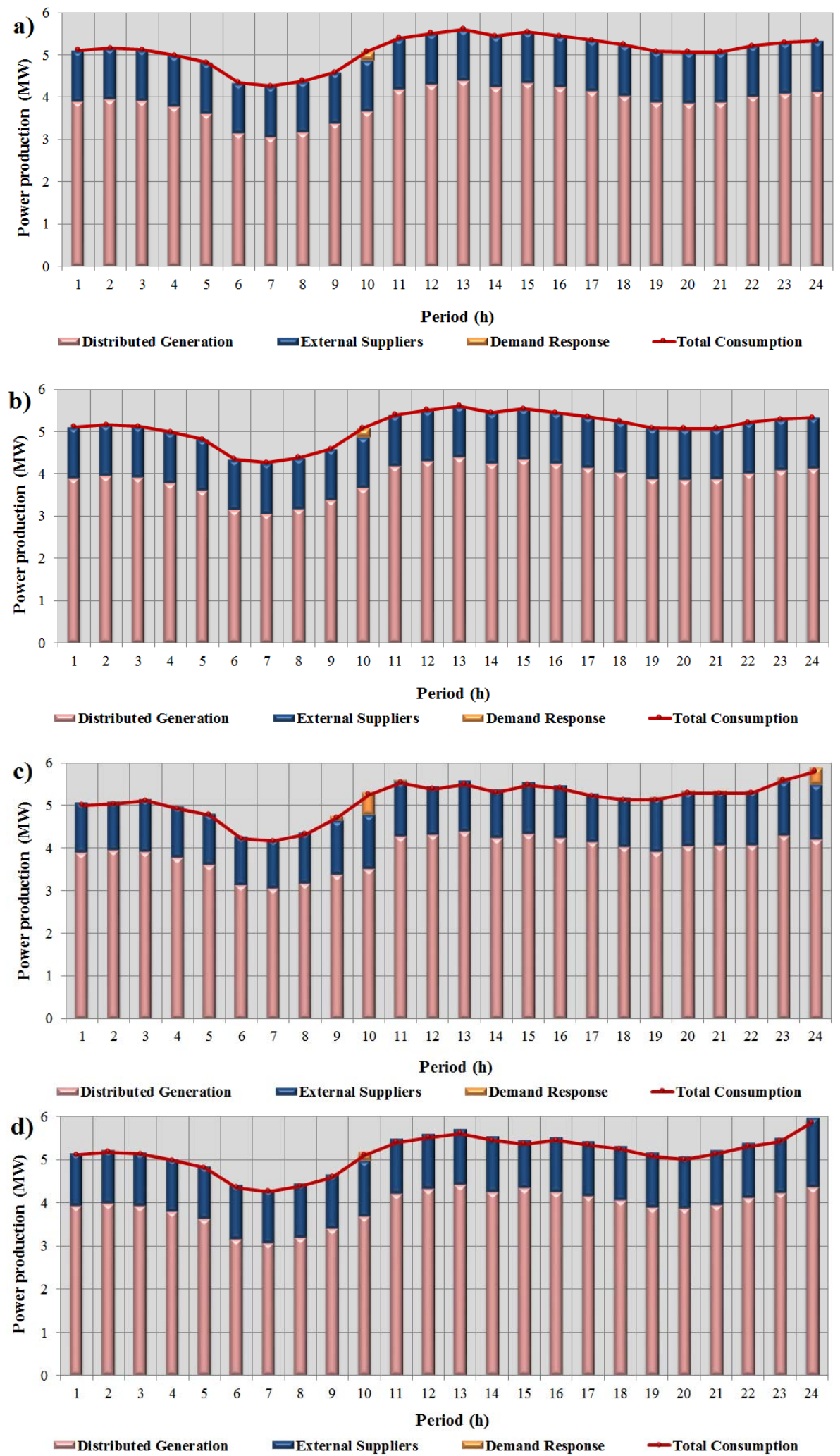

Fig. 6. Generation scheduling: a) MINLP b) MILP c) DSA d) HDSA (top-to-bottom). 


\subsection{0-bus network scenario}

In the 180-bus scenario the fleet of EVs considered was 6000 EVs. The total energy demand predicted with the tool for the trips was $34.26 \mathrm{MWh}$ corresponding to an average of $5.7 \mathrm{kWh}$ per vehicle and a total of 10137 trips (average of 1.7 trips per vehicle). The discharging cost considered for the EVs and ESS was set to 0.19 m.u. $/ \mathrm{kWh}$ and $0.18 \mathrm{~m} . \mathrm{u} . / \mathrm{kWh}$, respectively. It is assumed that the discharging price covered the battery degradation costs. A charging/discharging efficiency of $90 \%$ was considered. The external supplier contract is $10 \mathrm{MW}$, with a minimum of $2 \mathrm{MW}$ purchase in the considered time horizon. The distribution network representation can be seen in Fig. 7.

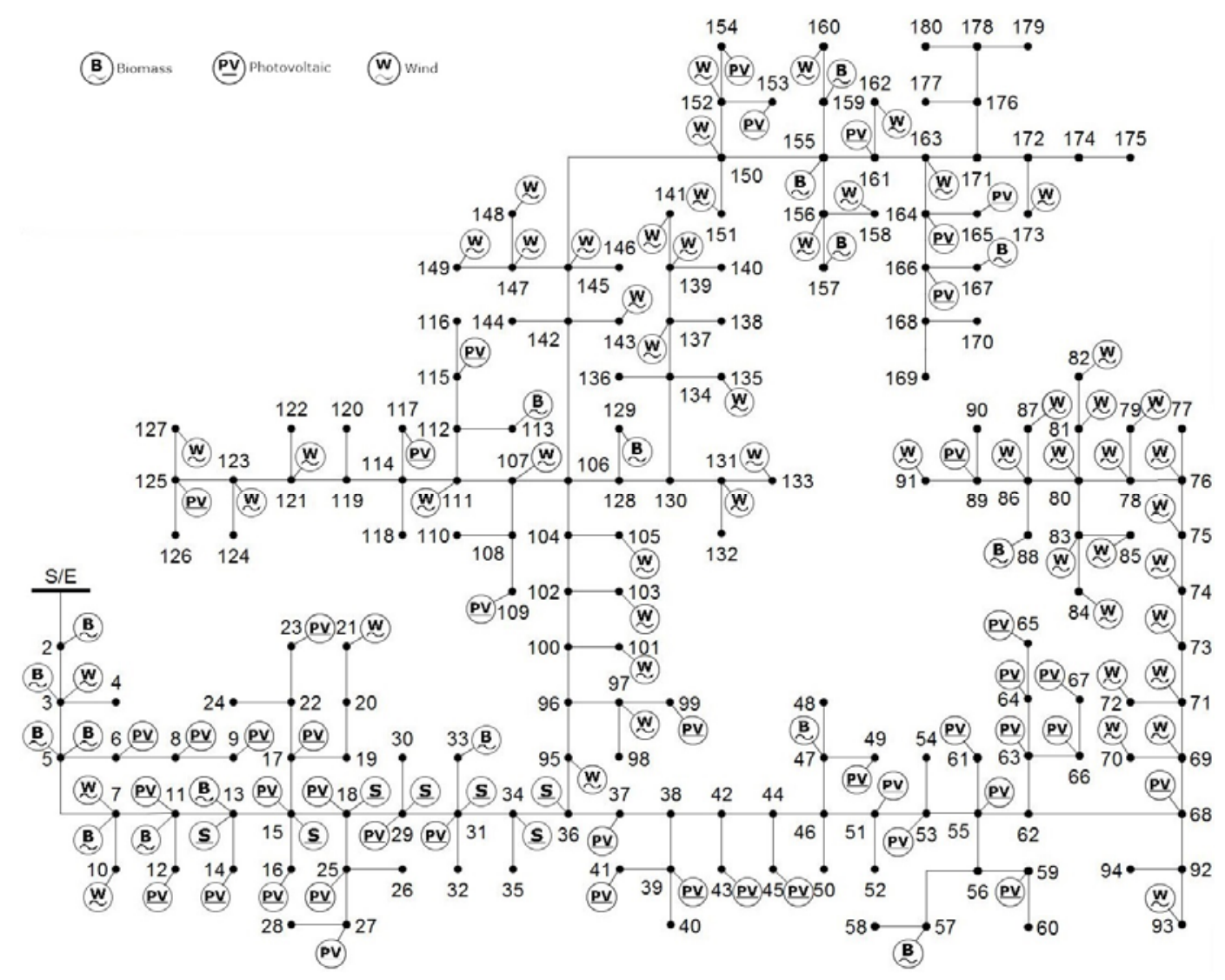

Fig. 7. Single-line diagram of 180 bus network.

Error! Reference source not found. presents the considered costs and the capacity of the system. The total forecasted regular load without EVs, ESS and DR was $243.36 \mathrm{MWh}$. 
Table 5. 180-bus grid scenario characterization

\begin{tabular}{|c|c|c|c|c|c|}
\hline Resources & $\begin{array}{l}\text { Min. price } \\
(\text { m.u./kWh })\end{array}$ & $\begin{array}{l}\text { Max. price } \\
(\text { m.u./kWh) }\end{array}$ & $\begin{array}{l}\text { Min. capacity } \\
(M W)\end{array}$ & $\begin{array}{l}\text { Max. capacity } \\
\text { (MW) }\end{array}$ & $\begin{array}{l}\text { Units } \\
\#\end{array}$ \\
\hline PV & 0.15 & 0.15 & 0.00 & 1.49 & 44 \\
\hline Wind & 0.09 & 0.09 & 1.07 & 1.76 & 55 \\
\hline Biomass & 0.13 & 0.13 & 1.98 & 1.98 & 17 \\
\hline $\begin{array}{l}\text { External } \\
\text { supplier }\end{array}$ & 0.10 & 0.16 & 10.00 & 10.00 & 1 \\
\hline V2G & 0.19 & 0.19 & 19.05 & 19.05 & 6000 \\
\hline ESS & 0.18 & 0.18 & 1.20 & 1.20 & 7 \\
\hline DR & 0.02 & 0.02 & 2.47 & 4.26 & 90 \\
\hline
\end{tabular}

For this scenario the parameters of the metaheuristics found in 33-bus scenario were used (see Table 2). A reduced set of methods and configurations were selected taken into account the previous tests with 33-bus scenario. The methods tested for 180-bus scenario were PSO, QPSO, DSA, HDSA with MD signaling, standard QPSO with linearly decreasing alpha, MILP and MINLP. Table 6 shows the results of the methods over 100 runs. The percentage of feasible solutions obtained with MD signaling over the 100 runs was $100 \%$ in PSO, QPSO and DSA methods while the standard QPSO with linearly decreasing alpha was $0 \%$ this time. The standard deviation was 17 m.u. for PSO and 9 m.u. for QPSO and DSA with MD signaling. Fig. 8 shows the box plot for the average results achieved in the performed tests, namely minimum, maximum, median, $75^{\text {th }}(\mathrm{Q} 3)$ and $25^{\text {th }}$ (Q1) percentile for each metaheuristic. Standard QPSO without MD signaling is not included in this figure because all the solutions obtained were infeasible. Moreover, HDSA achieved the same result in all run tests and therefore a standard deviation of 0 m.u., hence, not showing in the box plot.

The MILP approach obtained higher profits again in this scenario due to absence of power losses. After evaluating the MILP in the full AC power flow, the profit was corrected to 3072 m.u., but this solution included violations related with insufficient generation in some periods, which was caused by the additional power losses not evaluated by the MILP model. The MINLP considered the network equations but took more than 13 days to solve the optimization problem while metaheuristics took 24 minutes on average. Particularly, HDSA took only 150 seconds on average (with 100 iterations) with a profit $4 \%$ worse than MINLP but $10 \%$ higher than DSA. To evaluate the memory used by the applications the same procedure used for 33-bus scenario was done. In this 
case, metaheuristics presented an average peak memory use of $12 \mathrm{MB}$, MILP using TOMLAB was $164 \mathrm{MB}$ and HDSA peaked 164 MB. GAMS memory used was measured by analyzing windows memory information which was around $1080 \mathrm{MB}$.

Table 6. 180-bus scenario methods' results (averaged over 100 runs)

\begin{tabular}{c|c|c|c|c|c|c|c}
\hline \hline Method & $\begin{array}{c}\text { Changed } \\
\text { parameters }\end{array}$ & $\begin{array}{c}\text { MD } \\
\text { signaling }\end{array}$ & $\begin{array}{c}\text { Income } \\
(\boldsymbol{m} . u .)\end{array}$ & $\begin{array}{c}\text { Operation } \\
\text { Costs (m.u.) }\end{array}$ & $\begin{array}{c}\text { Profits } \\
(\boldsymbol{m} . u .)\end{array}$ & $\begin{array}{c}\text { Execution } \\
\text { time (s) }\end{array}$ & \#Iterations \\
\hline \hline PSO & & $\mathrm{Y}$ & 44,885 & 42,081 & 2803 & 1197 & 1342 \\
\hline QPSO & & $\mathrm{Y}$ & 45,312 & 42,439 & 2873 & 1628 & 1794 \\
\hline QPSO & $\alpha(1 \text { to } 0.3)^{2}$ & $\mathrm{~N}$ & \multicolumn{6}{|c}{$100 \%$ infeasible solutions } \\
\hline DSA & & $\mathrm{Y}$ & 45,391 & 42,469 & 2922 & 1384 & 1996 \\
\hline HDSA & Max. 100 it. & $\mathrm{Y}$ & 41,075 & 37,838 & 3237 & 150 & 100 \\
\hline MILP & & & 42,571 & 38,813 & 3758 & 76 & \\
\hline MINLP & & 42,303 & 38,938 & 3365 & $1,161,672$ & \\
\hline \hline
\end{tabular}

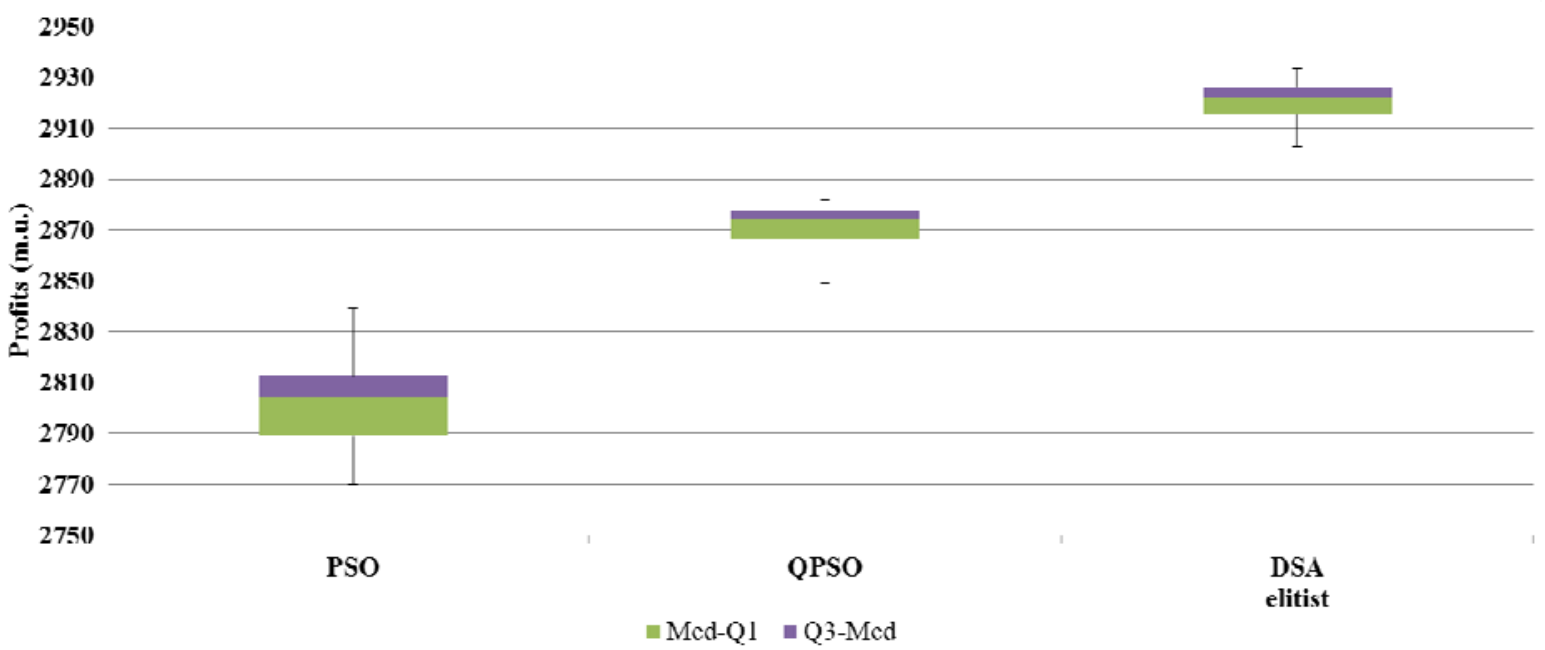

Fig. 8. Box plot for the average results of 100 runs in the 180-bus scenario.

Fig. 9 shows the average fitness values over 100 runs representing the convergence test with iterations fixed to 2000 for comparison purposes. The configurations presented in Table 6 that obtained positive profit (negative fitness) and $100 \%$ feasible solutions in all run tests were considered in this plot, namely PSO, QPSO and DSA with MD signaling. The analysis of the convergence reveals very similar performance as registered in the 33bus scenario. The metaheuristics with MD signaling presented very similar convergence properties with a fast evolution in the first few iterations and slower improvement onwards. In the same manner, DSA obtained slightly 
higher fitness on average when compared with QPSO and PSO while QPSO was slightly better than PSO but worse than DSA. In this scenario QPSO with linearly decreasing alpha was not able to provide feasible solutions in all run tests.

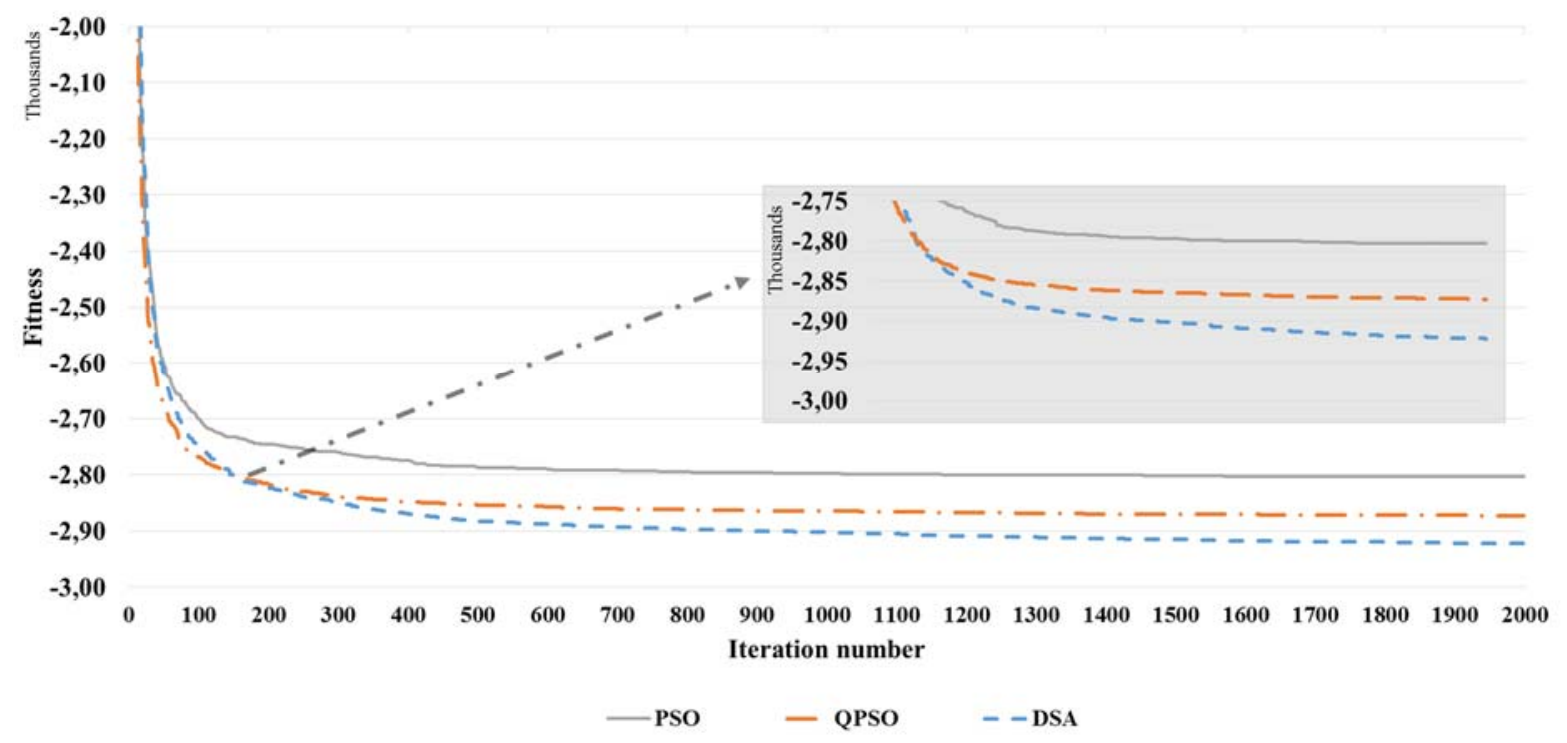

Fig. 9. Convergence test over 100 runs for selected metaheuristics: PSO, QPSO, DSA.

Table 7 shows the results of the ANOVA tests, which consisted in an individual ANOVA test for each comparison, namely DSA with other metaheuristics and HDSA with DSA. The tests indicate that the results obtained previously are significant and valid at the 0.01 significance level. Hence, the null hypothesis is rejected and the alternative hypothesis is accepted, i.e. the means are significantly different. It can be statistically concluded that HDSA is better than DSA and DSA is better than PSO and QPSO in $99 \%$ of the cases.

Table 7. - ANOVA test between the algorithms in the 180-bus scenario

\begin{tabular}{cccc}
\hline $\begin{array}{c}\text { Compared } \\
\text { algorithm }\end{array}$ & $\begin{array}{c}\text { DSA versus } \\
\text { PSO }\end{array}$ & $\begin{array}{c}\text { DSA versus } \\
\text { QPSO }\end{array}$ & HDSA versus DSA \\
\hline$\mu_{1}$ & 2922 & & 3237 \\
$\mu_{2}$ & 2803 & 2873 & 2922 \\
$\sigma_{1}$ & 9 & & 0 \\
$\sigma_{2}$ & 17 & 9 & 9 \\
F-value & 772 & 293 & 24031 \\
$p$-value & Infinitesimal & Infinitesimal & Infinitesimal \\
Significance & Very & Very significant & Extremely \\
& significant & significant \\
\hline
\end{tabular}

Fig. 10 and Fig. 11 presents the load and generation scheduling, respectively, as optimized by MINLP in GAMS, and HDSA ${ }^{3}$. The depicted results indicate a very different scheduling pattern (and a load diagram

\footnotetext{
${ }^{3}$ The complete results of the 180-bus scenario can be found in http://www.gecad.isep.ipp.pt/ies/public-data/swevo/
} 
profile) compared with the previous 33-bus scenario, e.g. selling energy was not scheduled but the use of EVs discharge was present in this case and DR use was higher. When comparing the results between MINLP and HDSA, it is clear that a very similar scheduling was achieved. The EVs charge was mostly scheduled during night periods when the energy from the suppliers was cheaper, corresponding to a total of 54.91 MWh in MINLP and 52.46 MWh in HDSA. In the 33-bus scenario some EVs charge was scheduled for the afternoon valley but in the 180 -bus scenario the valley is inexistent. Regarding generation, the external suppliers provided $54 \%$ and $56 \%$ of the total energy demand in MINLP (92.07 MWh) and HDSA (91.57 MWh). The DG played an important role with $31 \%$ of the total energy demand in both methods, namely $163.54 \mathrm{MWh}$ and $168.05 \mathrm{MWh}$ in MINLP and HDSA, respectively. The DR also played a significant role with a share of $10 \%$, namely $30.44 \mathrm{MWh}$ and 30.16 MWh in MINLP and HDSA, respectively. The EVs discharge corresponded to 5\% and 3\% in MINLP (14.81 MWh) and HDSA (9.13 MWh), respectively. The total active power losses were lower in MINLP (2.60 MWh) when compared with HDSA (3.09 MWh). In some periods the active power losses were very low because the local resources of the grid (DG, EVs, DR, etc.) reduce the power flow in the lines.
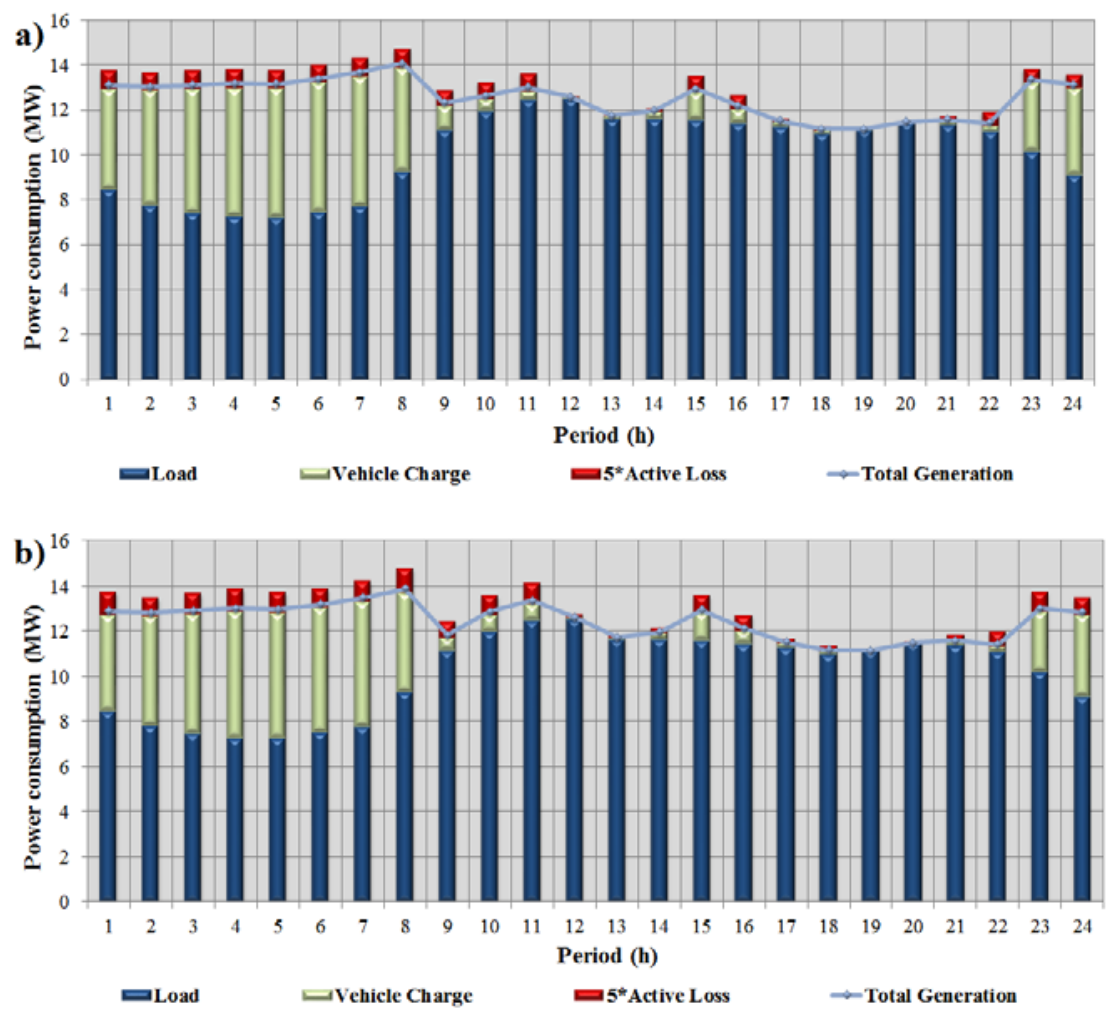

Fig. 10. Load scheduling: a) MINLP b) HDSA (top-to-bottom). 

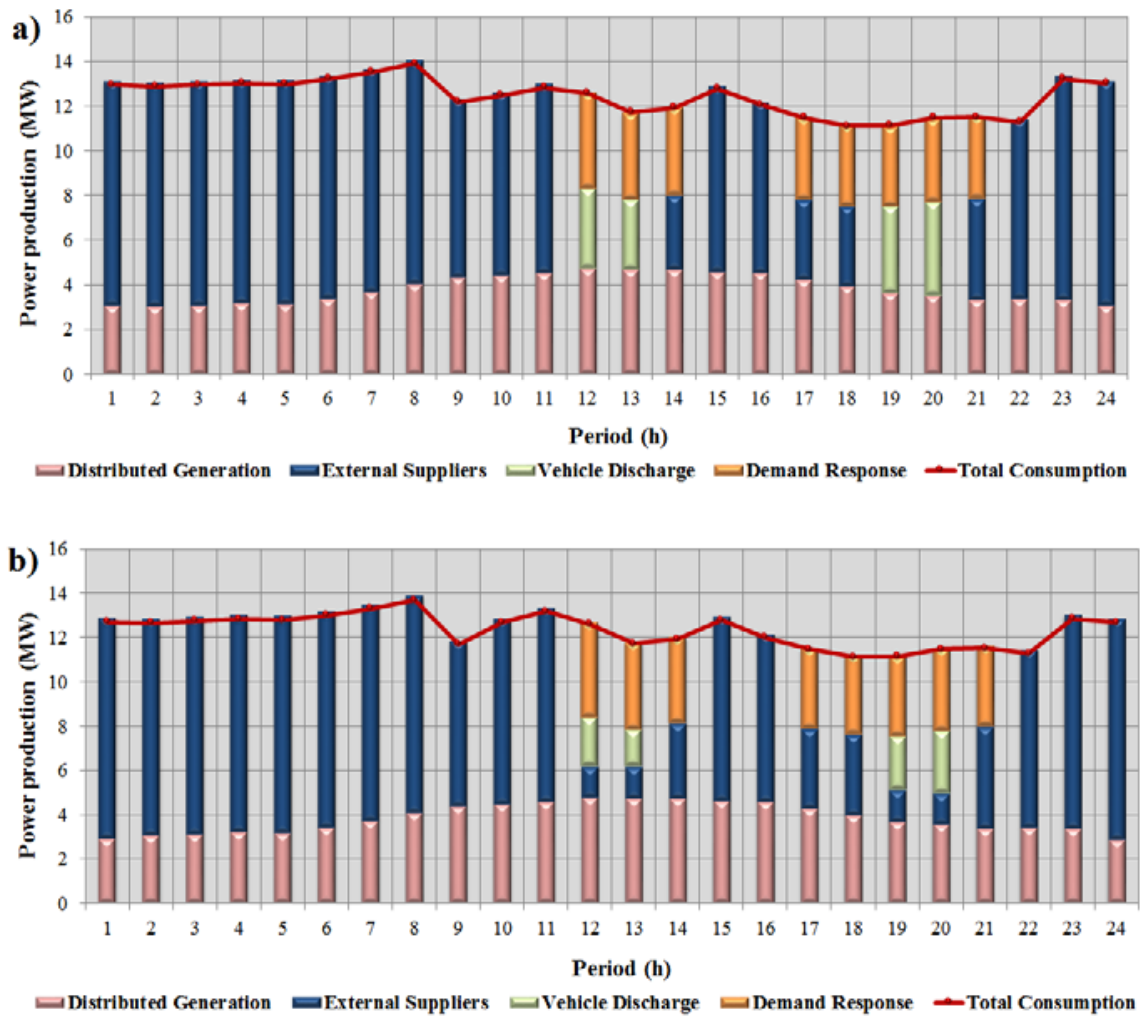

Fig. 11. Generation scheduling: a) MINLP b) HDSA (top-to-bottom).

\subsection{Discussion of the results}

The effect of the MD signaling in the metaheuristics was very positive in the presented cases. The tests show a substantial improvement in terms of solution quality (fitness) and more importantly in obtaining feasible solutions when compared with the standard versions (100\% feasible in all cases). Without MD signaling only QPSO with linearly decreasing alpha was able to obtain $100 \%$ feasible solutions in all run tests for the 33-bus scenario. In the 180 -bus scenario it was not possible to obtain positive profit and $100 \%$ feasible solutions in standard QPSO, i.e. without MD signaling. The convergence test showed that it is possible to reduce computational effort by reducing execution time with a superior convergence rate with MD signaling. Furthermore, it seems that the MD signaling effect was independent of the metaheuristic because the convergence profile revealed similar performance in both scenarios. In fact, the results with MD signaling showed that on average DSA performed slightly better than PSO and QPSO but the differences noticed were relatively negligible. In spite of better results (profits) in the deterministic approaches, the excessive time took by MINLP method (13 days in the 180-bus scenario) is currently not acceptable for the problem under study in 
large-scale scenarios. By contrast, the deterministic MILP method presented an excellent execution time but fails to include the full AC network constraints which may result in an unrealistic solution in some circumstances. The advantage of HDSA was reducing execution time while achieving close results to MINLP reference, particularly in the 180-bus scenario as the standard metaheuristics without MD signaling have failed. The disadvantage of using the deterministic based approaches is that a good mathematical solver for large-scale problems is required. Good commercial solvers are available but can be expensive. An important aspect verified in the tested scenarios was the very low memory used by metaheuristics approaches, i.e. between $4 \mathrm{MB}$ and 12 MB while deterministic approaches used that amount several fold (between 52-1080 MB). There were slightly differences in the power flow and consequently in the power losses between the MINLP and metaheuristics approaches, which is related with the iterative power flow approach used in metaheuristics and the method represented by constraints (4)-(7). Hence, adjusting the obtained MINLP results by running a post-optimization power flow revealed a slightly increase in the power losses and a decrease in the overall profit. This seems to close the gap between the test methods and the reference MINLP, e.g. HDSA to less than $1 \%$ difference in terms of objective function.

\section{Conclusions}

A new approach is presented in this paper to solve optimization problems with a population-based metaheuristics approach, known as multi-dimensional signaling. The problem under study in this work is a largescale ERM problem in SGs, considering several resources, namely DR programs, DG units, EVs and ESS from the perspective of a VPP. The results show that the standard metaheuristics struggle to obtain feasible and good solutions. The findings of this paper are summarized as follows:

- The standard metaheuristics without MD signaling have presented the following feasibility results: $60 \%$ in standard PSO, $0 \%$ in QPSO with fixed alpha, $100 \%$ in QPSO with linearly decreasing alpha, $15 \%$ in DSA elitist and $85 \%$ in DSA bijective for the 33-bus scenario while $0 \%$ feasible solutions in the 180-bus scenarios.

- The MD signaling approach allowed to obtain 100\% feasible solutions for all the metaheuristics in the run tests and in both test scenarios. 
- The MD signaling obtained better objective function (profits) compared to standard metaheuristics. For instance $23 \%$ higher in 33-bus scenario, and all the standard metaheuristics presented violations in 180-bus scenario .

- The deterministic approaches demonstrated to be more resource intensive. They need more system' memory (52-1080 MB) in large-scale problems, compared to metaheuristics that need less memory capacity (4-12 MB).

- Combining a linear formulation using MILP (relaxed version of MINLP) with the metaheuristics techniques, such as the HDSA, can provide significant reduction in execution time and better objective function. However, a good solver is required for large-scale problems as well as more computing requirements.

It might be possible that in the future, several methods like the ones presented run the ERM problems in a parallel platform simultaneous providing better decision support in adequate time, thus enabling better choices to decision makers in the operation of smart grids.

\section{Acknowledgements}

The present work was done and funded in the scope of the following projects: EUREKA - ITEA2 Project SEAS with project number 12004 and GID-MicroRede, project $\mathrm{n}^{\circ} 34086$, co-funded by COMPETE under FEDER via QREN; PTDC/SEN-ENR/122174/2010; UID/EEA/00760/2013 and SFRH/BD/87809/2012 funded by FEDER Funds through COMPETE program and by National Funds through FCT.

$\begin{array}{ll}\text { Nomenclature } & \\ \text { Indices } & \\ I & \text { DG units } \\ t & \text { time periods } \\ b & \text { buses } \\ L & \text { loads } \\ S & \text { external suppliers } \\ V & \text { EVs } \\ E & \text { ESSs } \\ M & \text { energy buyers } \\ \text { Sets } & \\ \Omega_{D G}^{d} & \text { set of dispatchable DG units } \\ \Omega_{D G}^{n d} & \text { set of non dispatchable DG units } \\ \Omega_{D G}^{b} & \text { set of DG units connected at bus } b \\ \Omega_{v}^{b, t} & \text { set of EVs at bus } b \text { during time period } t\end{array}$




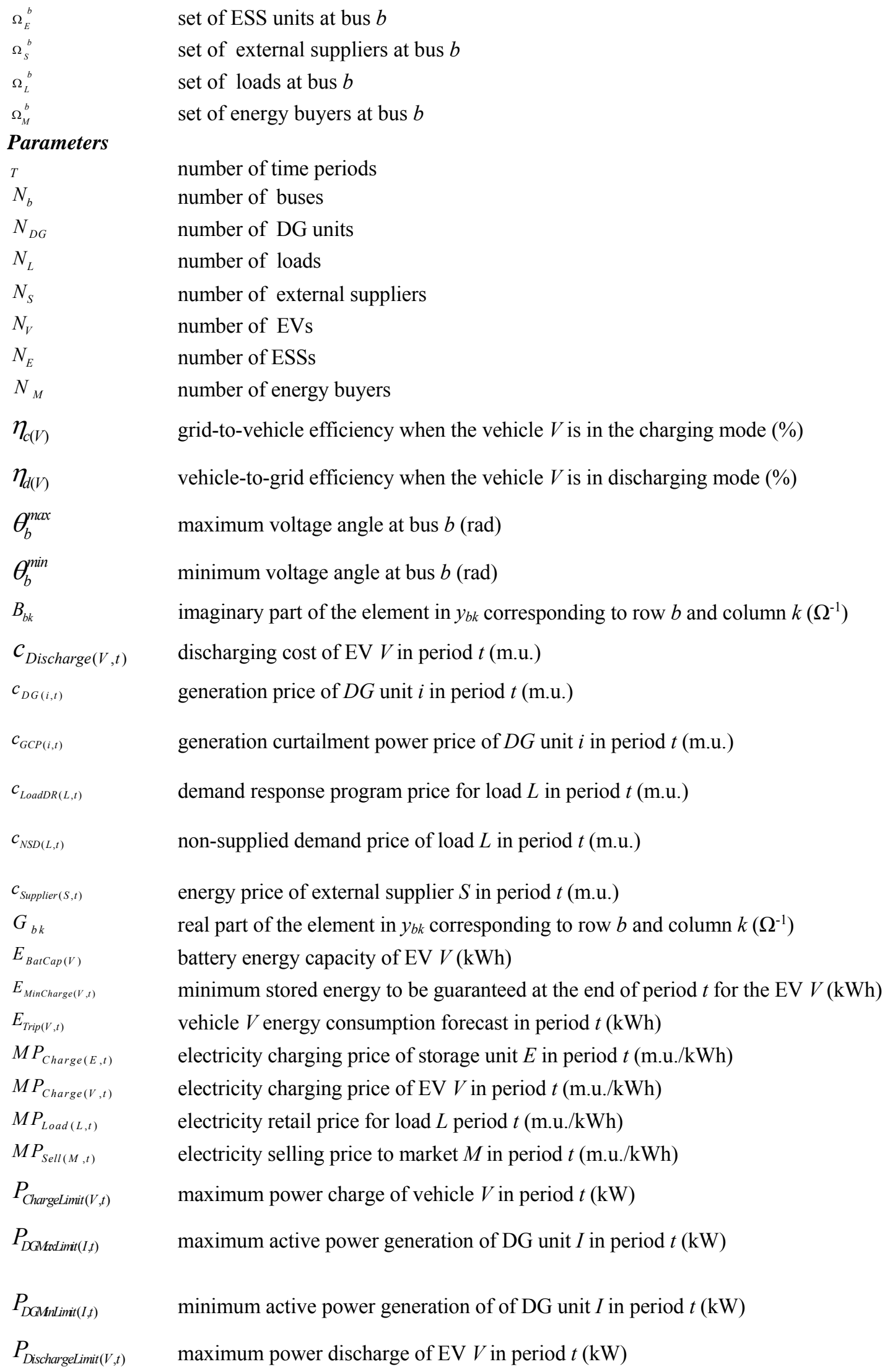




\begin{tabular}{|c|c|}
\hline$P_{L o a d(L, t)}$ & day-ahead active power load forecast of load $L$ in period $t(\mathrm{~kW})$ \\
\hline$P_{\text {LoadDRMaxLimit }(L, t)}$ & maximum active power reduce permitted for load $L$ in period $t(\mathrm{~kW})$ \\
\hline$P_{\text {Sipplierlinit }(S, t)}$ & maximum active power of upstream supplier $S$ in period $t(\mathrm{~kW})$ \\
\hline Qremadini(t,, ) & maximum reactive power generation of distributed generator unit $D G$ in period $t$ (kvar) \\
\hline$Q_{D G M n L i m i t(i, t)}$ & minimum reactive power generation of distributed generator unit $D G$ in period $t$ (kvar) \\
\hline$Q_{\mathrm{cod}(L, t)}$ & day-ahead reactive power load forecast of load $L$ in period $t$ (kvar) \\
\hline$Q_{\text {spplierlinit }(S, t)}$ & maximum reactive power of upstream supplier $S$ in period $t$ (kvar) \\
\hline$S_{b k}^{\max }$ & maximum apparent power flow established in line that connected buses $b$ and $k(\mathrm{kVA})$ \\
\hline$S_{T F R_{-} H V / M V(b)}^{\max }$ & maximum apparent power in HV/MV power transformer connected to bus $b(\mathrm{kVA})$ \\
\hline$S_{T F R \_M V / L V(b)}^{\max }$ & maximum apparent power in MV/LV power transformer connected to bus $b(\mathrm{kVA})$ \\
\hline$S_{b k}^{\max }$ & maximum apparent power flow established in line that connected buses $b$ and $k(\mathrm{kVA})$ \\
\hline$V_{b}^{\max }$ & maximum voltage magnitude at bus $b$ (p.u.) \\
\hline$V_{b}^{\text {in }}$ & minimum voltage magnitude at bus $b$ (p.u.) \\
\hline$y_{b k}$ & admittance of line that connect buses $b$ and $k\left(\Omega^{-1}\right)$ \\
\hline$y_{\text {Shunt_b}}$ & shunt admittance of line connected to bus $b\left(\Omega^{-1}\right)$ \\
\hline$\Delta t$ & duration of time period $t$ (hours) \\
\hline
\end{tabular}

Variables

$O C_{T o t a l}^{D+1}$

total day-ahead operation cost (m.u.)

$I_{\text {Total }}^{D+1}$

total day-ahead income (m.u.)

$\theta_{b(t)}$

voltage angle at bus $b$ in period $t(\mathrm{rad})$

$E_{\text {Stored }(E, t)}$

energy stored in ESS unit $E$ at the end of period $t(\mathrm{kWh})$

$E_{\text {Stored }(V, t)}$

energy stored in EV $V$ at the end of period $t(\mathrm{kWh})$

$P_{\text {Charge(E,t) }}$

power charge of ESS $E$ in period $t(\mathrm{~kW})$

$P_{\text {Charge }(V, t)}$

power charge of vehicle $V$ in period $t(\mathrm{~kW})$

$P_{D G(I, t)}$

active power generation of DG unit $I$ in period $t(\mathrm{~kW})$

$P_{\text {Discharge }(E, t) \quad \text { power discharge of ESS } E \text { in period } t(\mathrm{~kW})}$

$P_{\text {Discharge }(V, t) \quad \text { power discharge of EV } V \text { in period } t(\mathrm{~kW})}$

$P_{G C P(I, t)} \quad$ generation curtailment power of $D G$ unit $I$ in period $t(\mathrm{~kW})$ 


\begin{tabular}{|c|c|}
\hline$P_{\operatorname{LoadDR}(L, t)}$ & $\begin{array}{l}\text { active power reduction of load } L \text { in period } t \text { due to the demand response program } \\
\text { implementation }(\mathrm{kW})\end{array}$ \\
\hline$P_{N S D(L, t)}$ & non-supplied demand for load $L$ in period $t(\mathrm{~kW})$ \\
\hline$P_{\operatorname{Sell}(M, t)}$ & sell to market $M$ in period $t(\mathrm{~kW})$ \\
\hline$P_{\text {Spplier }(S, t)}$ & active power flow in the branch connecting to external supplier $S$ in period $t(\mathrm{~kW})$ \\
\hline$P_{\text {TFR_HV/M }(b, t)}$ & active power in HV/MV power transformer connected in bus $b$ to period $t(\mathrm{~kW})$ \\
\hline$P_{T F R_{-} M / L V(b, t)}$ & active power in MV/LV power transformer connected in bus $b$ to period $t(\mathrm{~kW})$ \\
\hline$Q_{D G(I, t)}$ & reactive power generation of DG unit $I$ in period $t$ (kvar) \\
\hline$Q_{\text {Supplier }(S, t)}$ & reactive power flow in the branch connecting to supplier $S$ in period $t$ (kvar) \\
\hline$Q_{T F R_{-} H V / M V(b, t)}$ & reactive power in HV/MV power transformer connected in bus $b$ in period $t$ (kvar) \\
\hline$Q_{T F R_{-} M V / L V(b, t)}$ & reactive power in $\mathrm{MV} / \mathrm{LV}$ power transformer connected in bus $b$ in period $t$ (kvar) \\
\hline$V_{b(t)}$ & voltage magnitude at bus $b$ in period $t$ (p.u.) \\
\hline$X_{(E, t)}$ & $\begin{array}{l}\text { binary decision variable for power discharging of ESS E in period } t \text { ( } 1 \text { if the ESS is } \\
\text { charging and } 0 \text { otherwise) }\end{array}$ \\
\hline$X_{(V, t)}$ & $\begin{array}{l}\text { binary decision variable for power discharging of } \mathrm{EV} \mathrm{V} \text { in period } \mathrm{t} \text { ( } 1 \text { if the } \mathrm{EV} \text { is charging } \\
\text { and } 0 \text { otherwise) }\end{array}$ \\
\hline$X_{D Q(i, t)}$ & $\begin{array}{l}\text { binary decision variable for the commitment status of DG unit } I \text { in period } t \text { ( } 1 \text { if the unit is } \\
\text { online and } 0 \text { otherwise) }\end{array}$ \\
\hline$Y_{(E, t)}$ & $\begin{array}{l}\text { binary decision variable for power charging of ESS } E \text { in period } t \text { ( } 1 \text { if the ESS is charging } \\
\text { and } 0 \text { otherwise) }\end{array}$ \\
\hline$Y_{(V, t)}$ & $\begin{array}{l}\text { binary decision variable for power charging of } \mathrm{EV} V \text { in period } t \text { ( } 1 \text { if the } \mathrm{EV} \text { is charging } \\
\text { and } 0 \text { otherwise) }\end{array}$ \\
\hline
\end{tabular}

\section{References}

[1] N. Balac, “Green Machine” Intelligence: Greening and Sustaining Smart Grids, Intell. Syst. IEEE. 28 (2013) 50-55. doi:10.1109/MIS.2013.127.

[2] A.Y. Saber, G.K. Venayagamoorthy, Intelligent unit commitment with vehicle-to-grid-A cost-emission optimization, J. Power Sources. 195 (2010) 898-911. doi:DOI 10.1016/j.jpowsour.2009.08.035.

[3] A.Y. Saber, G.K. Venayagamoorthy, Resource Scheduling Under Uncertainty in a Smart Grid with Renewables and Plug-in Vehicles, IEEE Syst. J. 6 (2012) 103-109. doi:10.1109/jsyst.2011.2163012.

[4] J. Soares, H. Morais, Z. Vale, Particle Swarm Optimization based approaches to vehicle-to-grid scheduling, in: 2012 IEEE Power Energy Soc. Gen. Meet., IEEE, 2012: pp. 1-8. doi:10.1109/PESGM.2012.6345358.

[5] J. Soares, T. Sousa, H. Morais, Z. Vale, P. Faria, An optimal scheduling problem in distribution networks considering V2G, 2011 IEEE Symp. Comput. Intell. Appl. Smart Grid CIASG. (2011) 1-8. doi:10.1109/CIASG.2011.5953342.

[6] T.. Sousa, H.. Morais, Z.. Vale, P.. Faria, J.. Soares, Intelligent Energy Resource Management Considering Vehicle-to-Grid: A Simulated Annealing Approach, IEEE Trans. Smart Grid, Spec. Issue Transp. Electrif. Veh. Appl. (2012).

[7] T. Sousa, H. Morais, J. Soares, Z. Vale, Day-ahead resource scheduling in smart grids considering Vehicle-to-Grid and network constraints, Appl. Energy. 96 (2012) 183-193. doi:10.1016/j.apenergy.2012.01.053. 
[8] M. Motevasel, A.R. Seifi, Expert energy management of a micro-grid considering wind energy uncertainty, Energy Convers. Manag. 83 (2014) 58-72. doi:10.1016/j.enconman.2014.03.022.

[9] A. Chaouachi, R.M. Kamel, R. Andoulsi, K. Nagasaka, Multiobjective Intelligent Energy Management for a Microgrid, IEEE Trans. Ind. Electron. 60 (2013) 1688-1699. doi:10.1109/TIE.2012.2188873.

[10] G.J. Osório, E.M.G. Rodrigues, J.M. Lujano-Rojas, J.C.O. Matias, J.P.S. Catalão, New control strategy for the weekly scheduling of insular power systems with a battery energy storage system, Appl. Energy. 154 (2015) 459-470. doi:10.1016/j.apenergy.2015.05.048.

[11] A. Zakariazadeh, S. Jadid, P. Siano, Integrated operation of electric vehicles and renewable generation in a smart distribution system, Energy Convers. Manag. 89 (2015) 99-110. doi:10.1016/j.enconman.2014.09.062.

[12] M. Honarmand, A. Zakariazadeh, S. Jadid, Integrated scheduling of renewable generation and electric vehicles parking lot in a smart microgrid, Energy Convers. Manag. 86 (2014) 745-755. doi:10.1016/j.enconman.2014.06.044.

[13] W. Su, J. Wang, J. Roh, Stochastic Energy Scheduling in Microgrids With Intermittent Renewable Energy Resources, IEEE Trans. Smart Grid. 5 (2014) 1876-1883. doi:10.1109/TSG.2013.2280645.

[14] L. Ruey-Hsun, L. Jian-Hao, A Fuzzy-Optimization Approach for Generation Scheduling With Wind and Solar Energy Systems, Power Syst. IEEE Trans. 22 (2007) 1665-1674. doi:10.1109/TPWRS.2007.907527.

[15] C. Binding, D. Gantenbein, B. Jansen, O. Sundstroem, P.B. Andersen, F. Marra, et al., Electric Vehicle Fleet Integration in the Danish EDISON Project - A Virtual Power Plant on the Island of Bornholm, 2010. $<$ Go to ISI $>$ ://WOS:000287611902065.

[16] M. Vasirani, R. Kota, R.L.G. Cavalcante, S. Ossowski, N.R. Jennings, An Agent-Based Approach to Virtual Power Plants of Wind Power Generators and Electric Vehicles, IEEE Trans. Smart Grid. 4 (2013) 1314-1322. doi:10.1109/TSG.2013.2259270.

[17] M. Silva, H. Morais, Z. Vale, P. Faria, Short-term Scheduling Considering Five-minute and Hourahead Energy Resource Management, 2012 Ieee Power Energy Soc. Gen. Meet. (2012). $<$ Go to ISI $>: / /$ WOS:000312493706083.

[18] P. Faria, Z. Vale, J. Soares, J. Ferreira, Demand Response Management in Power Systems Using a Particle Swarm Optimization Approach, Intell. Syst. IEEE. PP (2011) 1.

[19] Q. Wu, Grid Integration of Electric Vehicles in Open Electricity Markets, John Wiley \& Sons, 2013.

[20] G.K. Venayagamoorthy, Dynamic, Stochastic, Computational and Scalable Technologies for Smart Grids, Ieee Comput. Intell. Mag. 6 (2011) 22-35. doi:Doi 10.1109/Mci.2011.941588.

[21] M. Stadler, M. Kloess, M. Groissboeck, G. Cardoso, R. Sharma, M.C. Bozchalui, et al., Electric storage in California's commercial buildings, Appl. Energy. 104 (2013) 711-722.

doi:10.1016/j.apenergy.2012.11.033.

[22] Z. Jian-hua, BYD EV charging challenges solutions, in: Power Electron. Syst. Appl. (PESA), 2011 4th Int. Conf., IEEE, 2011: pp. 1-4.

[23] S. Burer, A.N. Letchford, Non-convex mixed-integer nonlinear programming: A survey, Surv. Oper. Res. Manag. Sci. 17 (2012) 97-106. doi:10.1016/j.sorms.2012.08.001.

[24] Z. Wan, G. Wang, B. Sun, A hybrid intelligent algorithm by combining particle swarm optimization with chaos searching technique for solving nonlinear bilevel programming problems, Swarm Evol. Comput. 8 (2013) 26-32. doi:10.1016/j.swevo.2012.08.001.

[25] T. Berthold, Heuristic algorithms in global MINLP solvers, Verlag Dr. Hut, 2014.

[26] S. Caron, G. Kesidis, Incentive-Based Energy Consumption Scheduling Algorithms for the Smart Grid, Smart Grid Commun. (SmartGridComm), 2010 First IEEE Int. Conf. (2010) 391-396. doi:10.1109/SMARTGRID.2010.5622073.

[27] I. Koutsopoulos, L. Tassiulas, Control and Optimization Meet the Smart Power Grid - Scheduling of Power Demands for Optimal Energy Management, Energy. (2010) 9. doi:10.1145/2318716.2318723. 
[28] N. Hassan, M. Pasha, C. Yuen, S. Huang, X. Wang, Impact of Scheduling Flexibility on Demand Profile Flatness and User Inconvenience in Residential Smart Grid System, Energies. 6 (2013) 66086635. doi:10.3390/en6126608.

[29] M. Gendreau, J.-Y. Potvin, Handbook of Metaheuristics, 2010. doi:10.1007/978-1-4419-1665-5.

[30] P. Siarry, Special Issue: Advances in metaheuristics for hard optimization: new trends and case studies, Eng. Appl. Artif. Intell. 23 (2010) 633-634. doi:DOI 10.1016/j.engappai.2010.03.003.

[31] S. Voss, Parallel metaheuristics: A new class of algorithms, J. Oper. Res. Soc. 59 (2008) 871. < Go to ISI $>: / / 000255502700018$.

[32] I. Boussaïd, J. Lepagnot, P. Siarry, A survey on optimization metaheuristics, in: Inf. Sci. (Ny)., 2013: pp. 82-117. doi:10.1016/j.ins.2013.02.041.

[33] P. Civicioglu, Transforming geocentric cartesian coordinates to geodetic coordinates by using differential search algorithm, Comput. Geosci. 46 (2012) 229-247. doi:DOI 10.1016/j.cageo.2011.12.011.

[34] J. Soares, T. Sousa, H. Morais, Z. Vale, B. Canizes, A. Silva, Application-Specific Modified Particle Swarm Optimization for energy resource scheduling considering vehicle-to-grid, Appl. Soft Comput. 13 (2013) 4264-4280. doi:DOI 10.1016/j.asoc.2013.07.003.

[35] J. Sun, C.H. Lai, X.J. Wu, Particle Swarm Optimisation: Classical and Quantum Perspectives, Taylor \& Francis, 2011. http://books.google.pt/books?id=EEES0b11RB0C.

[36] B. Liu, Composite Differential Search Algorithm, J. Appl. Math. (2014). http://www.hindawi.com/journals/jam/2014/294703/abs/ (accessed May 15, 2015).

[37] J. Soares, M. Silva, T. Sousa, Z. Vale, H. Morais, Distributed energy resource short-term scheduling using Signaled Particle Swarm Optimization, Energy. 42 (2012) 466-476. doi:DOI 10.1016/j.energy.2012.03.022.

[38] Y. del Valle, G.K. Venayagamoorthy, S. Mohagheghi, J.C. Hernandez, R.G. Harley, Particle swarm optimization: Basic concepts, variants and applications in power systems, Ieee Trans. Evol. Comput. 12 (2008) 171-195. doi:Doi 10.1109/Tevc.2007.896686.

[39] TOMLAB, TOMLAB optimization, (2015). http://tomopt.com/tomlab/about/.

[40] GAMS, GAMS, (2015). http://www.gams.com/ (accessed April 13, 2015).

[41] S.Y.S. You, C. Traeholt, B. Poulsen, A market-based Virtual Power Plant, in: 2009 Int. Conf. Clean Electr. Power, IEEE, 2009: pp. 460-465. doi:10.1109/ICCEP.2009.5212012.

[42] P.B. Andersen, B. Poulsen, M. Decker, C. Traeholt, J. Ostergaard, Evaluation of a Generic Virtual Power Plant framework using service oriented architecture, in: 2008 IEEE 2nd Int. Power Energy Conf., IEEE, 2008: pp. 1212-1217. doi:10.1109/PECON.2008.4762651.

[43] M. Peik-herfeh, H. Seifi, M.K. Sheikh-El-Eslami, Two-stage approach for optimal dispatch of distributed energy resources in distribution networks considering virtual power plant concept, Int. Trans. Electr. Energy Syst. 24 (2014) 43-63. doi:10.1002/etep.1694.

[44] E.A. Setiawan, Concept and Controllability of Virtual Power Plant, Kassel University, 2007.

[45] J. Soares, Z. Vale, H. Morais, Decision support tool for Virtual Power Players: Hybrid Particle Swarm Optimization applied to Day-ahead Vehicle-To-Grid Scheduling, ISAP. (2013).

[46] A. Munawar, M. Wahib, M. Munetomo, K. Akama, Solving Extremely Difficult MINLP Problems Using Adaptive Resolution Micro-GA with Tabu Search, in: Learn. Intell. Optim., Springer, 2011: pp. 203-217. doi:10.1007/978-3-642-25566-3_15.

[47] B.W. Wah, Y. Chen, Solving large-scale nonlinear programming problems by constraint partitioning, in: Princ. Pract. Constraint Program. 2005, Springer, 2005: pp. 697-711.

[48] G.P. Rangaiah, Stochastic global optimization: techniques and applications in chemical engineering, World Scientific, 2010.

[49] A. Zakariazadeh, S. Jadid, P. Siano, Multi-objective scheduling of electric vehicles in smart 
distribution system, Energy Convers. Manag. 79 (2014) 43-53. doi:10.1016/j.enconman.2013.11.042.

[50] R. Poli, Analysis of the Publications on the Applications of Particle Swarm Optimisation, J. Artif. Evol. Appl. 2008 (2008) 1-10. doi:10.1155/2008/685175.

[51] M.R. AlRashidi, M.E. El-Hawary, A Survey of Particle Swarm Optimization Applications in Electric Power Systems, IEEE Trans. Evol. Comput. 13 (2009) 913-918. doi:10.1109/TEVC.2006.880326.

[52] S. Sivasubramani, Economic Operation of Power Systems Using Hybrid Optimization Techniques, Indian Institute of Technology Madras, 2011.

[53] M. Joorabian, E. Afzalan, Optimal power flow under both normal and contingent operation conditions using the hybrid fuzzy particle swarm optimisation and Nelder-Mead algorithm (HFPSO-NM), Appl. Soft Comput. 14, Part C (2014) 623-633. doi:http://dx.doi.org/10.1016/j.asoc.2013.09.015.

[54] J. Kennedy, R. Eberhart, Particle swarm optimization, 1995 Ieee Int. Conf. Neural Networks Proceedings, Vols 1-6. (1995) 1942-1948. <Go to ISI>://A1995BF46H00374.

[55] V. Miranda, H. Keko, A. Jaramillo, EPSO: Evolutionary particle swarms, Stud. Comput. Intell. 66 (2007) 139-167. doi:10.1007/978-3-540-72377-6_6.

[56] C.-C. Chang, J. Tsai, S.-J. Pei, Quantum particle swarm optimisation algorithm for feedback control of semi-autonomous driver assistance systems, IET Intell. Transp. Syst. 8 (2014) 608-620. doi:10.1049/iet-its.2012.0204.

[57] Y. Fu, M. Ding, C. Zhou, H. Hu, Route Planning for Unmanned Aerial Vehicle (UAV) on the Sea Using Hybrid Differential Evolution and Quantum-Behaved Particle Swarm Optimization, IEEE Trans. Syst. Man, Cybern. Syst. 43 (2013) 1451-1465. doi:10.1109/TSMC.2013.2248146.

[58] P. Civicioglu, Transforming geocentric cartesian coordinates to geodetic coordinates by using differential search algorithm, Comput. Geosci. 46 (2012) 229-247. doi:DOI 10.1016/j.cageo.2011.12.011.

[59] V. Trianni, E. Tuci, K.M. Passino, J.A.R. Marshall, Swarm Cognition: an interdisciplinary approach to the study of self-organising biological collectives, Swarm Intell. 5 (2011) 3-18. doi:DOI 10.1007/s11721-010-0050-8.

[60] D. Thukaram, H.M. Wijekoon Banda, J. Jerome, A robust three phase power flow algorithm for radial distribution systems, Electr. Power Syst. Res. 50 (1999) 227-236. doi:10.1016/S0378-7796(98)001503.

[61] M.E. Baran, F.F. Wu, Network Reconfiguration in Distribution-Systems for Loss Reduction and Load Balancing, IEEE Trans. Power Deliv. 4 (1989) 1401-1407. doi:Doi 10.1109/61.25627.

[62] A. Zervos, C. Lins, J. Muth, RE-thinking 2050: a 100\% renewable energy vision for the European Union, EREC, 2010.

[63] J. Soares, B. Canizes, C. Lobo, Z. Vale, H. Morais, Electric Vehicle Scenario Simulator Tool for Smart Grid Operators, Energies. 5 (2012) 1881-1899. doi:10.3390/en5061881. 